\title{
Notes on the solutions of Zamolodchikov-type recursion relations in Virasoro minimal models
}

\author{
Nina Javerzat, ${ }^{a}$ Raoul Santachiara ${ }^{a}$ and Omar Foda ${ }^{b}$ \\ ${ }^{a}$ LPTMS, CNRS, Université Paris-Sud, Université Paris-Saclay, \\ 91405 Orsay, France \\ ${ }^{b}$ School of Mathematics and Statistics, University of Melbourne, \\ Parkville, Victoria 3010, Australia \\ E-mail: Nina.Javerzat@lptms.u-psud.fr, \\ Raoul. Santachiara@lptms.u-psud.fr, omar . foda@unimelb.edu . au
}

ABSTRACT: We study Virasoro minimal-model 4-point conformal blocks on the sphere and 0-point conformal blocks on the torus (the Virasoro characters), as solutions of Zamolodchikov-type recursion relations. In particular, we study the singularities due to resonances of the dimensions of conformal fields in minimal-model representations, that appear in the intermediate steps of solving the recursion relations, but cancel in the final results.

KEYwords: Conformal Field Theory, Lattice Integrable Models

ARXiv EPRINT: 1806.02790 


\section{Contents}

1 Introduction 1

1.1 The conformal bootstrap and Virasoro conformal blocks 1

1.2 Zamolodchikov's recursion relation 1

$\begin{array}{ll}1.3 \text { The singularities } & 1\end{array}$

1.4 The present work 2

1.5 Outline of contents 2

2 Virasoro algebra, representations and conformal blocks 2

2.1 The Virasoro algebra, generators and central charge 2

2.2 Verma modules 3

2.3 Degenerate representations 3

2.3.1 Null states vanish 3

2.3.2 Null states do not vanish 4

2.4 The Virasoro minimal models 4

2.4.1 Remark 4

2.5 The Virasoro conformal blocks 5

3 The 4-point conformal blocks on the sphere 5

3.1 The 4-point conformal block on the sphere 5

$\begin{array}{ll}3.2 & \text { The elliptic recursion relation } \\ 3.3 & \end{array}$

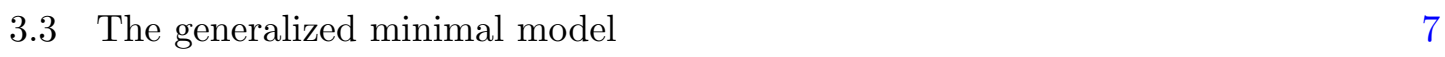

$\begin{array}{lll}\text { 3.3.1 The regularization } \epsilon & 7\end{array}$

$\begin{array}{ll}3.4 & \text { Minimal-model conformal blocks. }\end{array}$

$\begin{array}{lll}3.4 .1 & \text { The regularization } \epsilon^{\prime} & 8\end{array}$

3.5 Conjecture 8

3.6 Further example 8

4 1-point conformal blocks on the torus $\quad 9$

4.1 The 1-point conformal block on the torus 9

4.2 The recursion relation of Fateev and Litvinov [3] 9

4.2.1 Remark 9

$\begin{array}{lll}\text { 4.2.2 The recursion relation } & 10\end{array}$

$\begin{array}{lll}4.2 .3 & \text { The } R_{r, s}^{\text {tor }} \text { numerators } & 10\end{array}$

$\begin{array}{ll}4.2 .4 \text { Examples } & 10\end{array}$

$\begin{array}{lll}4.3 \text { Remark } & 10\end{array}$

5 The 0-point functions on the torus: the characters 11

5.1 General central charge and general $\Delta_{\text {int }} \quad 11$

5.2 General central charge, $\Delta_{\text {int }}=\Delta_{m, n} \quad 11$

$\begin{array}{lll}\text { 5.2.1 Internal field regularization } & 11\end{array}$ 
$\begin{array}{lll}5.3 & \text { Characters in minimal models } & 12\end{array}$

5.3.1 The regularization $\epsilon^{\prime \prime} \quad 12$

5.4 Example 1. The Ising model, $\left(p^{\prime}, p\right)=(4,3),(m, n)=(1,1) \quad 13$

$\begin{array}{lll}5.5 & \text { General case } & 14\end{array}$

$\begin{array}{llr}6 & \text { Conclusions } & 18\end{array}$

$\begin{array}{ll}\text { A A direct computation at } c=-2 & 19\end{array}$

B About $R_{m, n}^{\text {tor }} \quad 21$

\section{Introduction}

\subsection{The conformal bootstrap and Virasoro conformal blocks}

In spite of the enormous progress in our understanding of $2 \mathrm{D}$ conformal field theories over the past 35 years, important classes of two-dimensional conformal field theories remain to be discovered, or at least better-understood. An outstanding example is critical percolation, which is known to be a CFT with Virasoro central charge $c=0$, but the correlation functions of this CFT remain to be computed. Recently, new numerical 2D conformal bootstraps that fully exploit the full 2D local conformal symmetries were developed and new 2D CFT's were discovered $[8,12]$. These 2D bootstraps are based on Virasoro conformal blocks, and the corresponding solutions are functions of infinite-dimensional representations of local conformal transformations, as opposed to bootstraps that are valid in any dimension, which are based on global conformal blocks, and the corresponding solutions are functions of finite-dimensional representations of global conformal transformations.

\subsection{Zamolodchikov's recursion relation}

To implement the new 2D bootstraps numerically, one needs to compute the 4-point conformal blocks on the sphere efficiently. The most efficient known method to compute 4-point conformal blocks on the sphere is Zamolodchikov's recursion relation. In fact, solving the $2 \mathrm{D}$ bootstrap efficiently is what motivated Al. Zamolodchikov to develop the recursion relations in the first place $[14,15]$. There are two versions of Zamolodchikov's recursion relation, a hypergeometric version [14], and an elliptic version [15]. The elliptic version is particularly efficient, and will be the focus of the present work, and to fully understand this recursion relation, we will find it useful to consider a related recursion relation for the 1-point conformal block on the torus, and its 0-point conformal block limit, which is a Virasoro character.

\subsection{The singularities}

The 4-point conformal block on the sphere is a function of six parameters: the Virasoro central charge, the conformal dimension of the Virasoro representation that flows in the internal channel, and the conformal dimensions of the four external fields. The solution of 
the elliptic recursion relation displays a rich structure of poles. These poles are physical in the sense that they correspond to the propagation of states for suitable choices of the central charge and conformal dimensions. In a numerical 2D bootstrap based on Virasoro conformal blocks that are computed using the elliptic recursion relation, one must deal with these poles when exploring the space of possible crossing-symmetric CFT solutions. This happens, for example, in studies of percolation in the 2D Ising model [9]. When the central charge is such that one deals with minimal-model conformal blocks, additional poles appear. These additional poles are non-physical and appear due to resonances of conformal dimensions (see equation (2.11)) at rational values of the central charge. This complication requires a careful study of the pole structure of the elliptic recursion relation in the case of Virasoro minimal models, which is the aim of the present work.

\subsection{The present work}

We study the cancellation of the non-physical poles in computations of minimal-model conformal blocks using Zamolodchikov's elliptic recursion relation for the 4-point conformal block on the sphere. But the 4-point conformal block on the sphere is not the only or the simplest conformal block that can be computed using a recursion relation. In 2009, Poghossian [10], and independently Fateev and Litvinov [3] proposed recursion relations to compute Liouville 1-point conformal blocks on the torus. These recursion relations are equivalent [6], and we use the Fateev-Litvinov version to study minimal-model 1-point functions on the torus, and their 0-point limits (when the vertex operator insertion is the identity) which are Virasoro minimal-model characters, as the simplest examples of solutions of a Zamolodchikov-type elliptic recursion relation.

\subsection{Outline of contents}

In section 2, we recall basic facts related to the Virasoro algebra, its representations, and conformal blocks. In section 3, we consider the 4-point conformal blocks on the sphere as solutions of the recursion relation, study their singularities and their behaviour in the context of the Virasoro generalized minimal models and minimal models. In section 4, we consider the 1-point conformal block on the torus as solutions of the Fateev-Litvinov recursion relation. In section 5, we study the solutions of the Fateev-Litvinov recursion relations for the Virasoro minimal-model 1-point functions on the torus in the special case where the inserted vertex operator is the identity and the 1-point function reduces to the character of the irreducible highest-weight representation that flows in the torus. In appendix A, we include the details of an explicit computation, and in appendix B, we include technical details related to coefficients that appear in the recursion relations.

\section{Virasoro algebra, representations and conformal blocks}

We recall basic definitions related to the Virasoro algebra, representation theory, and conformal blocks. We refer the reader to the review [13].

\subsection{The Virasoro algebra, generators and central charge}

A Virasoro CFT is based on the Virasoro algebra,

$$
\left[L_{n}, L_{m}\right]=(n-m) L_{n+m}+\frac{c}{12} n\left(n^{2}-1\right) \delta_{n+m, 0}, \quad n, m \in \mathbb{Z},
$$


where the Virasoro generators $L_{n}, n \in \mathbb{Z}$ are the modes of the stress-energy tensor and $c$ is the central charge. The Liouville parametrization of the central charge is,

$$
c=1+6 Q^{2}, \quad Q=b+b^{-1}, \quad c \in \mathbb{C}
$$

\section{$2.2 \quad$ Verma modules}

Given a highest-weight state $|\Delta\rangle$, with highest weight $\Delta, L_{0}|\Delta\rangle=\Delta|\Delta\rangle$, the descendant states $L_{-n_{1}} \cdots L_{-n_{N}}|\Delta\rangle, n_{1} \geqslant n_{2} \geqslant \cdots \geqslant n_{N}$, form a basis of the Verma module $\mathcal{V}_{\Delta}$. A general element in this basis is $L_{-Y}|\Delta\rangle$, labeled by a Young diagram $Y=\left(n_{1}, \cdots, n_{N}\right)$, that has $N$ non-zero parts, and,

$$
L_{0}\left|L_{-Y} \Delta\right\rangle=(\Delta+|Y|)\left|L_{-Y} \Delta\right\rangle,
$$

where $|Y|=\sum_{i=1}^{N} n_{i}$ is the number of cells in the Young diagram $Y$. Using the statefield correspondence, we use $\Phi_{\Delta}(x)$ for the primary field of conformal dimension $\Delta$, and $L_{-Y} \Phi_{\Delta}(x)$ for the descendant fields. We parametrize the conformal dimension $\Delta$ by the parameter $Q,(2.2)$, and the charge $\alpha$,

$$
\Delta=\alpha(Q-\alpha)
$$

\subsection{Degenerate representations}

A degenerate representation has a highest weight $\Delta_{m, n}$,

$$
\Delta_{m, n}=\alpha_{m, n}\left(Q-\alpha_{m, n}\right), \quad \alpha_{m, n}=-\frac{1}{2}(m-1) b-\frac{1}{2}(n-1) b^{-1},
$$

and has a null state $\left|\chi_{m n}\right\rangle$ at level $m n,\left\langle\chi_{m n} \mid \chi_{m n}\right\rangle=0$. When a representation with highest weight $\Delta_{m n}$ appears in the spectrum of a given CFT model, two situations can occur.

\subsubsection{Null states vanish}

The corresponding representation module $\mathcal{V}_{m, n}$ is the quotient of a reducible Verma module by a non-trivial submodule,

$$
\mathcal{V}_{m n}=\frac{\mathcal{V}_{\Delta_{m n}}}{\mathcal{V}_{\Delta_{m n}+m n}}
$$

The representations $\mathcal{V}_{m n}$ form the spectrum of the Virasoro generalized minimal as well as the minimal models. The vanishing of the null state implies the fusion rules. The fusions of products of $\mathcal{V}_{m n}$ have simple expressions in the parametrization (2.4). For instance, the fusion of $\mathcal{V}_{m n}$ with a Verma module $\mathcal{V}_{\alpha}$ is a sum of $m n$ Verma modules and takes the form,

$$
\mathcal{V}_{m n} \times \mathcal{V}_{\alpha}=\sum_{i=\frac{1}{2}(1-m)}^{\frac{1}{2}(m-1)} \sum_{j=\frac{1}{2}(1-n)}^{\frac{1}{2}(n-1)} \mathcal{V}_{\alpha+i b+j b^{-1}}
$$

where the sums are in steps of 1. 


\subsubsection{Null states do not vanish}

Representations with non-vanishing null-states appear in Liouville field theory at $c \leqslant 1$ [12], and in computations of probabilities of non-local critical objects such as the left-right passage probability of an SLE interface. We will not deal with this case in the present work.

\subsection{The Virasoro minimal models}

$\mathcal{M}_{p p^{\prime}}$, are labeled by two positive co-prime integers $p$ and $p^{\prime}$, such that $0<p<p^{\prime}$. The space of chiral states of $\mathcal{M}_{p p^{\prime}}$ is generated by a Virasoro algebra with central charge,

$$
b=\left(-\frac{p^{\prime}}{p}\right)^{\frac{1}{2}}
$$

The space of chiral states splits into (typically finitely-many) fully-degenerate irreducible highest-weight modules $\mathcal{V}_{m n}$ labeled by two integers $m$ and $n$, such that $0<m<p$, and $0<n<p^{\prime}$. From (2.5), $\Delta_{m n}$ satisfies the negation relation, and the periodicity relation,

$$
\Delta_{m n}=\Delta_{-m,-n}, \quad \Delta_{m, n}=\Delta_{m+p, n+p^{\prime}}
$$

which combine to give,

$$
\Delta_{m, n}=\Delta_{m^{\prime}, n^{\prime}}, \quad m^{\prime}=p-m, \quad n^{\prime}=p^{\prime}-n,
$$

as well as an infinite chain of relations that involve 'resonant' conformal dimensions,

$$
\Delta_{m, n}=\Delta_{p+m, p^{\prime}+n}=\cdots
$$

Two pairs of indexes $(m, n)$ and $(r, s)$ are resonant if there are linked by a finite chain of transformations (2.9). In this case we use the notation,

$$
(m, n) \leftrightarrow(r, s)_{l}^{ \pm}
$$

to indicate that there exists an integer $l$ such that,

$$
(m, n) \leftrightarrow(r, s)_{l}^{ \pm} \Longrightarrow r=l p \pm m, \quad s=l p^{\prime} \pm n, \quad l \in \mathbb{N}
$$

\subsubsection{Remark}

In our notation, $0<p<p^{\prime}$, and $b=\sqrt{-p^{\prime} / p}$ is pure imaginary such that $|b|>0$. One can think of $|b|$ as the magnitude of the positive screening charge $\alpha_{+}>0$. We normally take the negative screening charge $\alpha_{-}<0$, and the background charge,

$$
-2 \alpha_{0}=-\left(\alpha_{+}+\alpha_{-}\right)
$$

that is, the background charge can be screened by the sum of a single $\alpha_{+}$and a single $\alpha_{-}$. 


\subsection{The Virasoro conformal blocks}

The conformal blocks are special functions of the Virasoro representations. We consider the 4-point conformal blocks on the sphere, the 1-point conformal blocks on the torus, and the 0-point conformal blocks on the torus, which are Virasoro characters. In all generality, these conformal blocks are defined in terms of the $p_{|Y|} \times p_{|Y|}$ matrix $S_{|Y|}\left(Y, Y^{\prime}\right)$ of inner products of descendants at level $|Y|$, where $p_{|Y|}$ is the number of partitions of $|Y|$,

$$
S_{|Y|}\left(Y, Y^{\prime}\right)=\left\langle L_{-Y} \Delta \mid L_{-Y^{\prime}} \Delta\right\rangle, \quad|Y|=\left|Y^{\prime}\right|,
$$

and the matrix elements,

$$
\left\langle L_{-Y_{1}} \Delta_{1}\left|L_{-Y_{2}} \Phi_{\Delta_{2}}(1)\right| L_{-Y_{3}} \Delta_{3}\right\rangle
$$

In Virasoro CFT's, the Shapolavov matrix and the 3-point functions are completely determined by the Virasoro algebra (2.1). Note that this is not true anymore for more general conformal chiral algebras such as the $\mathcal{W}^{N}$ algebras $[1,2]$.

\section{The 4-point conformal blocks on the sphere}

We outline Zamolodchikov's computation of the 4-point conformal block on the sphere, and study its poles.

\subsection{The 4-point conformal block on the sphere}

Global conformal symmetry determines the dependences of four-point blocks on three of the four positions $z_{i}$, and we assume $\left(z_{i}\right)=(x, 0, \infty, 1)$. The conformal block is a function of six parameters, the central charge, the cross-ratio $x$, the conformal dimensions of the four external fields $\Delta_{i}, i=1, \cdots, 4$, and the conformal dimension of the representation that flows in the internal channel $\Delta_{\text {int }}$. In terms of the vertex-operators charges,

$$
\Delta_{i}=\alpha_{i}\left(Q-\alpha_{i}\right), \quad \Delta_{\text {int }}=\alpha_{\text {int }}\left(Q-\alpha_{\text {int }}\right)
$$

The 4-point conformal block on the sphere has an $x$-series expansion,

$$
\mathcal{B}(\boldsymbol{\Delta}, x)=x^{-\Delta_{1}-\Delta_{2}+\Delta_{i n t}}\left(1+\mathcal{B}_{1}(\boldsymbol{\Delta}) x+\mathcal{B}_{2}(\boldsymbol{\Delta}) x^{2}+\cdots\right),
$$

where $\boldsymbol{\Delta}$ for the set of external and internal conformal dimensions $\left(\Delta_{1}, \Delta_{2}, \Delta_{3}, \Delta_{4}, \Delta_{\text {int }}\right)$, and,

$$
\mathcal{B}_{|Y|}(\boldsymbol{\Delta})=\sum_{\substack{Y, Y^{\prime} \\|Y|=\left|Y^{\prime}\right|}} S_{|Y|}^{(-1)}\left(Y, Y^{\prime}\right)\left\langle\Delta_{2}\left|\Phi_{1}(1)\right| L_{-Y^{\prime}} \Delta_{\text {int }}\right\rangle\left\langle L_{-Y} \Delta_{\text {int }}\left|\Phi_{3}(1)\right| \Delta_{4}\right\rangle,
$$

where $Y^{\prime}$ is any Young diagram such that $\left|Y^{\prime}\right|=|Y|$, and $\Phi_{i}$ is a primary field of conformal dimension $\Delta_{i}$. 


\subsection{The elliptic recursion relation}

In [15], Zamolodchikov introduced an elliptic recursion relation of the same 4-point conformal blocks on the sphere. ${ }^{1}$ The recursion parameter $q$ is a function of $x$

$$
q=\frac{1}{16}\left(x+\frac{1}{2} x^{2}+\frac{21}{64} x^{3}+\frac{31}{128} x^{4}+\mathcal{O}\left(x^{5}\right)\right),
$$

which follows from inverting,

$$
x=\frac{\theta_{2}^{4}(q)}{\theta_{3}^{4}(q)},
$$

where $\theta_{2}(q)$ and $\theta_{3}(q)$ are Jacobi theta functions,

$$
\theta_{2}(q)=\sum_{n=-\infty}^{\infty} q^{(n+1 / 2)^{2}}, \quad \theta_{3}(q)=\sum_{n=-\infty}^{\infty} q^{n^{2}},
$$

The conformal blocks can be written as,

$$
\begin{aligned}
\mathcal{B}(\Delta, c, x)= & x^{\frac{c-1}{24}-\Delta_{1}-\Delta_{2}}(1-x)^{\frac{c-1}{24}-\Delta_{2}-\Delta_{3}}(16 q)^{\Delta_{\text {int }}-\frac{Q^{2}}{4}} \theta_{3}(q)^{3 Q^{2}-4\left(\Delta_{1}+\Delta_{2}+\Delta_{3}+\Delta_{4}\right)} \\
& \times \mathcal{H}^{\mathrm{sph}}\left(\boldsymbol{\Delta}_{\text {ext }}, \Delta_{\mathrm{int}}, c, x\right),
\end{aligned}
$$

where the elliptic variable $q$ and function $\theta_{3}(q)$ are defined in (3.4) and in (3.6) and we use $\boldsymbol{\Delta}_{\text {ext }}$ for the set of external dimensions $\left(\Delta_{1}, \Delta_{2}, \Delta_{3}, \Delta_{4}\right)$. The analytic structure of the function $\mathcal{H}^{\mathrm{sph}}\left(\boldsymbol{\Delta}_{\text {ext }}, \Delta_{\text {int }}, c, x\right)$ is manifest in the following expansion, ${ }^{2}$

$$
\mathcal{H}^{\mathrm{sph}}\left(\boldsymbol{\Delta}_{\mathrm{ext}}, \Delta_{\mathrm{int}}, c, x\right)=1+\sum_{r s \geqslant 1}(16 q)^{r s} \frac{R_{r, s}^{\mathrm{sph}}\left(\boldsymbol{\Delta}_{\mathrm{ext}}, c\right)}{\Delta_{\mathrm{int}}-\Delta_{r, s}} \mathcal{H}^{\mathrm{sph}}\left(\boldsymbol{\Delta}_{\mathrm{ext}}, \Delta_{r,-s}, c, x\right),
$$

where,

$$
R_{m, n}^{\mathrm{sph}}(\Delta, c)=\frac{1}{r_{m, n}} P_{m, n}\left(\Delta_{1}, \Delta_{2}\right) P_{m, n}\left(\Delta_{3}, \Delta_{4}\right)
$$

The factors $P_{m, n}$ carries all dependence in $R_{m, n}^{\mathrm{sph}}(\boldsymbol{\Delta})$ on the external conformal dimensions $\Delta_{i}, i=1, \cdots, 4$. It is convenient to parametrize the conformal dimensions in terms of the momenta $\lambda_{i}$ and $\lambda_{m, n}$,

$$
\Delta_{i}=\frac{c-1}{24}+\lambda_{i}^{2}, \quad \Delta_{m, n}=\frac{c-1}{24}+\lambda_{m, n}^{2}
$$

In terms of these variables, one has,

$$
\begin{aligned}
P_{m, n}\left(\Delta_{1}, \Delta_{2}\right) & =\prod_{\rho, \sigma}\left(\lambda_{1}+\lambda_{2}-\lambda_{\rho, \sigma}\right)\left(\lambda_{1}-\lambda_{2}-\lambda_{\rho, \sigma}\right) \\
\rho & =1-m, 3-m, \cdots, m-1, \quad \sigma=1-n, 3-n, \cdots, n-1
\end{aligned}
$$

The factor $r_{m, n}$ is given by,

$$
\begin{aligned}
r_{m, n} & =-\frac{1}{2} \prod_{\rho, \sigma} 2 \lambda_{\rho, \sigma}, \\
\rho & =1-m, 2-m, \cdots, m, \quad \sigma=1-n, 2-n, \cdots, n, \quad(\rho, \sigma) \neq(0,0),(m, n)
\end{aligned}
$$

${ }^{1}$ In [11], Poghossian extended Zamolodchikov's elliptic recursion relation to a class of $\mathcal{W}_{3}$ Toda 4-point conformal blocks on the sphere.

${ }^{2}$ Note that some closed form expression has been found in [7]. 


\subsection{The generalized minimal model}

When the central charge is non-rational, but a degenerate representation $\mathcal{V}_{\Delta_{m, n}}$ flows in the channel, the recursion relation (3.8) has a pole related to the presence of a null-state at level $m n$ in $\mathcal{V}_{\Delta_{m, n}}$, and the corresponding Shapovalov matrix has a vanishing eigenvalue that produces the singularity in the expansion (3.3).

\subsubsection{The regularization $\epsilon$}

We introduce a regularization parameter $\epsilon$,

$$
\Delta_{\text {int }}=\Delta_{m, n}^{(\epsilon)}=\Delta_{m n}+\epsilon
$$

The limit $\epsilon \rightarrow 0$ in (3.8) exists only if the polynomial $P_{m, n}\left(\Delta_{1}, \Delta_{2}\right)$ and/or $P_{m, n}\left(\Delta_{3}, \Delta_{4}\right)$, defined in (3.9) and in (3.11), vanish. Recall that $P_{m, n}\left(\Delta_{1}, \Delta_{2}\right)$ vanishes when $\left(\mathcal{V}_{\Delta_{1}}, \mathcal{V}_{\Delta_{2}}, \mathcal{V}_{m, n}\right)$ satisfy the fusion rules (2.7), that is to say when $\alpha_{2}=\alpha_{1}+i b+j b^{-1}$, with $i \in\{(1-m) / 2,(3-m) / 2, \cdots,(m-1) / 2\}$ and $j \in\{(1-n) / 2,(3-m) / 2, \cdots,(n-1) / 2\}$. The generalized minimal model has a non-rational central charge, $c \notin \mathbb{Q}$, and a spectrum formed by all the degenerate representations $\mathcal{V}_{m, n}$ with $(m, n) \in \mathbb{N}_{+}$. All the fields in the spectrum satisfy the fusion rules (2.7), imposed by the condition $\chi_{m n}=0$. The conformal blocks of the generalized minimal model can be obtained by using the recursion relation with a simple limiting procedure. This consists in setting,

$$
\Delta_{i}=\Delta_{r_{i}, s_{i}}^{\left(\epsilon_{i}\right)}=\Delta_{r_{i}, s_{i}}+\epsilon_{i}, \quad i=1, \cdots, 4,
$$

with $\epsilon_{i} \rightarrow 0, i=1, \cdots, 4$ of the same order of $\epsilon, \epsilon_{i}=\mathcal{O}(\epsilon)$, and take the limit $\epsilon \rightarrow 0$. Using,

$$
\frac{H_{m, n}^{\mathrm{sph}}(\Delta, c, x)}{\Delta_{\mathrm{int}}-\Delta_{m, n}} \propto \frac{P_{m, n}\left(\Delta_{r_{1}, s_{1}}^{(\epsilon)} \Delta_{r_{2}, s_{2}}^{\left(\epsilon_{2}\right)}\right) P_{m, n}\left(\Delta_{r_{3}, s_{3}}^{\left(\epsilon_{3}\right)} \Delta_{r_{4}, s_{4}}^{\left(\epsilon_{4}\right)}\right)}{\Delta_{m, n}^{\epsilon}-\Delta_{m, n}} \sim O(\epsilon),
$$

it is straightforward to see that the term $\mathcal{H}_{m, n}^{s p h}(\boldsymbol{\Delta}, c, x)$ in (3.8) do not contribute. In the generalized minimal models therefore, the conformal block with $\Delta_{\text {int }}=\Delta_{m, n}$ is obtained using the sum in (3.8) where the term $(r, s)=(m, n)$ is omitted. We stress that, in this procedure limit, the final result is independent of the exact relation between the regularization parameters $\epsilon_{i}$ and $\epsilon$. The only thing that matters is the fact that the $\epsilon_{i}$ and $\epsilon$ are of the same order. As we will see later, this will not be the case for the computation of the characters.

\subsection{Minimal-model conformal blocks.}

We address here the problem of how to obtain the conformal blocks of minimal models $\mathcal{M}_{p, p^{\prime}}$ from the recursion relation (3.8). The main observation is that, with respect to the generalized minimal models, there are new poles appearing in (3.8). The location of these extra poles do not depend neither on the internal channel field nor on the external fields. They originate from the resonances in the conformal dimensions that occur when $c \in \mathbb{Q}$. 
Let us consider for instance one conformal block of the Ising minimal model, $\mathcal{M}_{4,3}$. At level 7, two terms appear,

$$
\frac{R_{2,1}^{\mathrm{sph}}\left(\boldsymbol{\Delta}_{\mathrm{ext}}, c\right)}{\Delta_{\mathrm{int}}-\Delta_{2,1}} \frac{R_{1,5}^{\mathrm{sph}}\left(\boldsymbol{\Delta}_{\mathrm{ext}}, c\right)}{\Delta_{2,-1}-\Delta_{1,5}}, \quad \text { and } \quad \frac{R_{1,3}^{\mathrm{sph}}\left(\boldsymbol{\Delta}_{\mathrm{ext}}, c\right)}{\Delta_{\mathrm{int}}-\Delta_{1,3}} \frac{R_{4,1}^{\mathrm{sph}}\left(\boldsymbol{\Delta}_{\mathrm{ext}}, c\right)}{\Delta_{-1,3}-\Delta_{4,1}},
$$

that are singular due to the fact that at $c=1 / 2,(2,-1) \rightarrow(1,5)^{-}$and $(-1,3) \rightarrow(4,1)^{-}$. Differently from the poles that originate when $\Delta_{\text {int }}=\Delta_{m, n}$, which are related to the nullstate at level $m n$, these other singularities can be considered an artifact of the recursion relation in the sense that they are not related to any special properties of descendant states. In the appendix we better explain this point with an explicit example.

\subsubsection{The regularization $\epsilon^{\prime}$}

In addition to (3.13), we introduce a regularization parameter $\epsilon^{\prime}$ to the central charge $c$,

$$
b^{2}=-\frac{p^{\prime}}{p}+\epsilon^{\prime}
$$

that is of the same order of $\epsilon, \epsilon^{\prime}=O(\epsilon)$.

\subsection{Conjecture}

We conjecture that, by setting (3.13), (3.14) and (3.17), the limit $\epsilon \rightarrow 0$ in the recursion relation (3.8) exists and provides the correct minimal model conformal block.

\subsection{Further example}

We have checked that the two terms (3.16) combine to give a finite contribution. Another example, at level 20, is the combination of the following five singular terms,

$$
\begin{aligned}
& \frac{R_{1,1}^{\mathrm{sph}}\left(\boldsymbol{\Delta}_{\mathrm{ext}}, c\right)}{\left(\Delta_{1,1}^{(\epsilon)}-\Delta_{1,1}\right)} \frac{R_{4,3}^{\mathrm{sph}}\left(\boldsymbol{\Delta}_{\mathrm{ext}}, c\right)}{\left(\Delta_{1,-1}-\Delta_{4,3}\right)} \frac{R_{7,1}^{\mathrm{sph}}\left(\boldsymbol{\Delta}_{\mathrm{ext}}, c\right)}{\left(\Delta_{4,-3}-\Delta_{7,1}\right)} \\
& +\frac{R_{1,1}^{\mathrm{sph}}\left(\boldsymbol{\Delta}_{\mathrm{ext}}, c\right)}{\left(\Delta_{1,1}^{(\epsilon)}-\Delta_{1,1}\right)} \frac{R_{2,5}^{\mathrm{sph}}\left(\boldsymbol{\Delta}_{\mathrm{ext}}, c\right)}{\left(\Delta_{1,-1}-\Delta_{2,5}\right)} \frac{R_{1,9}^{\mathrm{sph}}\left(\boldsymbol{\Delta}_{\mathrm{ext}}, c\right)}{\left(\Delta_{2,-5}-\Delta_{1,9}\right)} \\
& +\frac{R_{2,3}^{\mathrm{sph}}\left(\boldsymbol{\Delta}_{\mathrm{ext}}, c\right)}{\left(\Delta_{1,1}^{(\epsilon)}-\Delta_{2,3}\right)} \frac{R_{5,1}^{\mathrm{sph}}\left(\boldsymbol{\Delta}_{\mathrm{ext}}, c\right)}{\left(\Delta_{2,-3}-\Delta_{5,1}\right)} \frac{R_{1,9}^{\mathrm{sph}}\left(\boldsymbol{\Delta}_{\mathrm{ext}}, c\right)}{\left(\Delta_{5,-1}-\Delta_{1,9}\right)} \\
& +\frac{R_{2,3}^{\mathrm{sph}}\left(\boldsymbol{\Delta}_{\mathrm{ext}}, c\right)}{\left(\Delta_{1,1}^{(\epsilon)}-\Delta_{2,3}\right)} \frac{R_{1,7}^{\mathrm{sph}}\left(\boldsymbol{\Delta}_{\mathrm{ext}}, c\right)}{\left(\Delta_{2,-3}-\Delta_{1,7}\right)} \frac{R_{7,1}^{\mathrm{sph}}\left(\boldsymbol{\Delta}_{\mathrm{ext}}, c\right)}{\left(\Delta_{1,-7}-\Delta_{7,1}\right)}+\frac{R_{4,5}^{\mathrm{sph}}\left(\boldsymbol{\Delta}_{\mathrm{ext}}, c\right)}{\left(\Delta_{1,1}^{(\epsilon)}-\Delta_{4,5}\right)}
\end{aligned}
$$

to a finite contribution. If we can predict the singular terms that, at a given level, provide finite contributions, we have not been able to obtain a compact formula for these. As we will see in the following, we can control the contribution of these type of singularities in the computation of a simpler symmetry function, the character. 


\section{1-point conformal blocks on the torus}

We recall the Fateev-Litvinov recursion relation for the 1-point conformal block on the torus, and introduce the structure of its poles.

\subsection{The 1-point conformal block on the torus}

The Virasoro 1-point conformal block on the torus consists of a single vertex-operator insertion in a torus geometry, and a Virasoro irreducible highest-weight representation flows in the single internal channel of the torus. This is a function of four parameters, the central charge, the torus parameter $q$, the conformal dimension of the external field $\Delta_{\text {ext }}$, and the conformal dimension of the internal channel $\Delta_{\text {int }}$. The conformal dimension of the external vertex-operator is,

$$
\Delta_{\text {ext }}=\alpha_{\text {ext }}\left(Q-\alpha_{\text {ext }}\right)
$$

and similarly, the conformal dimension of the representation that flows in the torus is,

$$
\Delta_{\text {int }}=\alpha_{\text {int }}\left(Q-\alpha_{\text {int }}\right)
$$

The torus 1-point conformal block has the $q$-series expansion,

$$
\mathcal{F}(\boldsymbol{\Delta}, q)=1+\mathcal{F}_{1}(\boldsymbol{\Delta}) q+\mathcal{F}_{2}(\boldsymbol{\Delta}) q^{2}+\cdots,
$$

where $\boldsymbol{\Delta}$ is a pair of conformal dimensions $\left(\Delta_{\text {ext }}, \Delta_{\text {int }}\right)$, and,

$$
\mathcal{F}_{|Y|}(\boldsymbol{\Delta})=\sum_{\substack{Y, Y^{\prime} \\|Y|=\left|Y^{\prime}\right|}} S_{|Y|}^{(-1)}\left(Y, Y^{\prime}\right)\left\langle L_{-Y} \Delta_{\mathrm{int}}\left|\Phi_{\mathrm{ext}}(1)\right| L_{-Y^{\prime}} \Delta_{\mathrm{int}}\right\rangle
$$

\subsection{The recursion relation of Fateev and Litvinov [3]}

The 1-point conformal block on the torus is,

$$
\mathcal{F}(\boldsymbol{\Delta} \mid q)=\frac{q^{1 / 24}}{\eta(q)} \mathcal{H}(\boldsymbol{\Delta} \mid q),
$$

where,

$$
\frac{q^{1 / 24}}{\eta(q)}=\prod_{i=1}^{\infty} \frac{1}{1-q^{i}}=1+q+\cdots+p_{N} q^{N}+\cdots,
$$

$p_{N}$ is the number of partitions of $N \in \mathbb{N}$, and,

$$
\mathcal{H}(\boldsymbol{\Delta} \mid q)=\sum_{N=0}^{\infty} H_{N}\left(\alpha_{\text {ext }}, \Delta_{\text {int }}\right) q^{N}
$$

\subsubsection{Remark}

The factor $q^{1 / 24} / \eta(q)$ is the character of the Fock space of a free boson, and in (4.5), the 1-point function on the torus is written in terms of the free-boson of Feigin and Fuks $[4,5]$. This will become clear once we take the $\alpha_{\text {ext }} \rightarrow 0$ limit, and 1-point function becomes a character, in section 5 . 


\subsubsection{The recursion relation}

The recursion comes in the definition of $H_{N}$, the coefficients of the numerator of the conformal block,

$$
H_{N}\left(\alpha_{\text {ext }}, \Delta_{\text {int }}\right)=\sum_{r s=1}^{N} \frac{R_{r, s}^{\mathrm{tor}}\left(\alpha_{\mathrm{ext}}\right)}{\left(\Delta_{\mathrm{int}}-\Delta_{r, s}\right)} H_{N-r s}\left(\alpha_{\mathrm{ext}}, \Delta_{-r, s}\right), H_{0}\left(\alpha_{\mathrm{ext}}, \Delta_{\mathrm{int}}\right)=1
$$

\subsubsection{The $R_{r, s}^{\text {tor }}$ numerators}

$$
\begin{aligned}
R_{r, s}^{\mathrm{tor}}\left(\alpha_{\mathrm{ext}}\right) & =\frac{1}{4 r_{r, s}} \prod_{k} \prod_{l}\left(\frac{k-1}{2} b+\frac{l-1}{2} b^{-1}+\alpha_{\mathrm{ext}}\right), \\
k & =1-2 r, 3-2 r, \cdots, 2 r-1, \quad l=1-2 s, 3-2 s, \cdots, 2 s-1,
\end{aligned}
$$

$r_{r, s}$ is given by formula (3.12).

\subsubsection{Examples}

The simplest coefficients $H_{N}, N=1,2, \cdots$, in (4.7) are,

$$
\begin{aligned}
H_{1}\left(\alpha_{\text {ext }}, \Delta_{\text {int }}\right)= & \frac{R_{1,1}^{\text {tor }}\left(\alpha_{\text {ext }}\right)}{\left(\Delta_{\text {int }}-\Delta_{1,1}\right)}, \\
H_{2}\left(\alpha_{\text {ext }}, \Delta_{\text {int }}\right)= & \frac{R_{1,1}^{\text {tor }}\left(\alpha_{\text {ext }}\right)}{\left(\Delta_{\text {int }}-\Delta_{1,1}\right)} H_{1}\left(\alpha_{\text {ext }}, \Delta_{-1,1}\right) \\
& +\frac{R_{1,2}^{\text {tor }}\left(\alpha_{\text {ext }}\right)}{\left(\Delta_{\text {int }}-\Delta_{1,2}\right)}+\frac{R_{2,1}^{\text {tor }}\left(\alpha_{\text {ext }}\right)}{\left(\Delta_{\text {int }}-\Delta_{2,1}\right)}, \\
H_{3}\left(\alpha_{\text {ext }}, \Delta_{\text {int }}\right)= & \frac{R_{1,1}^{\text {tor }}\left(\alpha_{\text {ext }}\right)}{\left(\Delta_{\text {int }}-\Delta_{1,1}\right)} H_{2}\left(\alpha_{\text {ext }}, \Delta_{-1,1}\right)+\frac{R_{1,2}^{\text {tor }}\left(\alpha_{\text {ext }}\right)}{\left(\Delta_{\text {int }}-\Delta_{1,2}\right)} H_{1}\left(\alpha_{\text {ext }}, \Delta_{-1,2}\right) \\
& +\frac{R_{2,1}^{\text {tor }}\left(\alpha_{\text {ext }}\right)}{\left(\Delta_{\text {int }}-\Delta_{2,1}\right)} H_{1}\left(\alpha_{\text {ext }}, \Delta_{-2,1}\right)+\frac{R_{1,3}^{\text {tor }}\left(\alpha_{\text {ext }}\right)}{\left(\Delta_{\text {int }}-\Delta_{1,3}\right)}+\frac{R_{3,1}^{\text {tor }}\left(\alpha_{\text {ext }}\right)}{\left(\Delta_{\text {int }}-\Delta_{3,1}\right)}
\end{aligned}
$$

and so on.

\subsection{Remark}

Expanding (4.5), we obtain,

$$
\begin{aligned}
\mathcal{F}\left(\Delta_{\text {ext }}, \Delta_{\text {int }} \mid q\right) & =\left(H_{0}\right) q^{0}+\left(H_{0}+H_{1}\right) q+\left(2 H_{0}+H_{1}+H_{2}\right) q^{2}+\cdots \\
& =\sum_{N=0}^{\infty} \sum_{k=0}^{N} p(N-k) H_{k} q^{N}
\end{aligned}
$$

From (4.11), the structure of the conformal block $\mathcal{F}(\boldsymbol{\Delta} \mid q)$ is clear. In particular, if $\Delta_{\text {ext }}=$ 0 , and $H_{N}=0$, for all $N=1,2, \cdots$, we recover the character of the Fock space of a free boson, which is the character of a generic non-minimal conformal field theory. If $\Delta_{\text {ext }}=0$, and $H_{N}= \pm 1$, for appropriate values of $N$, null states and their descendants are removed and one obtains the character of an irreducible fully-degenerate highest-weight module. This will be discussed in detail in section 5 . 


\section{The 0-point functions on the torus: the characters}

We discuss the derivation of the character of the representation corresponding to $\Delta_{\text {int }}$ using the recursion relation and a particular limiting procedure, in three cases: $\mathbf{1}$. general central charge and general $\Delta_{\text {int }}, \mathbf{2}$. general central charge and degenerate representation $\Delta_{\text {int }}=\Delta_{m, n}$, and 3. minimal models $\mathcal{M}_{p, p^{\prime}}$ characters.

\subsection{General central charge and general $\Delta_{\text {int }}$}

We introduce regularization parameter $\epsilon$ that we set to 0 at the end. We set the inserted vertex-operator to be the identity, in the limit $\epsilon$ to zero,

$$
\alpha_{\mathrm{ext}}=2 \epsilon
$$

The factor 2 in the above definition is for convenience. For general $r, s \in \mathbb{N}$, the term,

$$
R_{r, s}^{\mathrm{tor}} \frac{\left(\alpha_{\mathrm{ext}}=2 \epsilon\right)}{\left(\Delta_{\mathrm{int}}-\Delta_{r, s}\right)}=-\epsilon \frac{\left(Q-2 \alpha_{r, s}\right)}{\left(\Delta_{\mathrm{int}}-\Delta_{r, s}\right)}+\mathcal{O}\left(\epsilon^{2}\right),
$$

vanishes in the limit $\epsilon \rightarrow 0$, provided that $\lim _{\epsilon \rightarrow 0} \Delta_{\text {int }} \neq \Delta_{r, s}$. All the $H_{i}$ are then zero and the expansion is given by (4.6). As expected, one finds the character $\chi_{\Delta_{\text {int }}}(q)$ of an irreducible Verma module of dimension $\Delta_{\text {int }}$.

\subsection{General central charge, $\Delta_{\text {int }}=\Delta_{m, n}$}

First we set $\alpha_{\text {ext }}=2 \epsilon$. Differently from the previous case, here we encounter the pole coming from the denominator $\left(\Delta_{\text {int }}-\Delta_{m, n}\right)$.

\subsubsection{Internal field regularization}

We need to regularize the dimension of the internal field, and we set,

$$
\alpha_{\mathrm{int}}=\alpha_{m, n}+\epsilon^{\prime},
$$

and we define,

$$
\Delta_{m, n}^{\left(\epsilon^{\prime}\right)}=\alpha_{\text {int }}\left(Q-\alpha_{\text {int }}\right)=\Delta_{m, n}+\epsilon^{\prime}\left(Q-2 \alpha_{m, n}\right)+\mathcal{O}\left(\epsilon^{\prime 2}\right)
$$

For $\epsilon, \epsilon^{\prime} \ll 1$, the term,

$$
\frac{R_{m, n}^{\mathrm{tor}}(2 \epsilon)}{\left(\Delta_{m, n}^{\left(\epsilon^{\prime}\right)}-\Delta_{m, n}\right)}=-\frac{\epsilon+\mathcal{O}\left(\epsilon^{2}\right)}{\epsilon^{\prime}+\mathcal{O}\left(\epsilon^{\prime 2}\right)}
$$

The result of the limit $\left(\epsilon, \epsilon^{\prime}\right) \rightarrow(0,0)$ depends therefore on the way one reaches the point $\left(\epsilon, \epsilon^{\prime}\right)=(0,0)$. For instance, if one first sends $\epsilon \rightarrow 0$ and then $\epsilon^{\prime} \rightarrow 0$, all the $H_{i}$ are zero and the character of a general Verma module is found. This result can be interpreted by saying that the null-state at level $\mathrm{nm}$ is not vanishing. Interestingly, such representation appears for instance in the construction of the Liouville theory for $c \leqslant 1$ [12]. By setting $\epsilon^{\prime}=\epsilon$ one finds instead that,

$$
\lim _{\epsilon \rightarrow 0} \frac{R_{m, n}^{\mathrm{tor}}\left(\alpha_{\mathrm{ext}}=2 \epsilon\right)}{\left(\Delta_{m, n}^{(\epsilon)}-\Delta_{m, n}\right)}=-1,
$$


for general $b$. The contribution (5.6) is the only non-zero term in $H_{m n}$, which is itself the only non-zero $H_{i}$. This contribution $H_{m n}=-1$ at level $m n$ corresponds to removing the null state. From equation (4.11), you can see that the expansion is (keeping only the non-zero terms),

$$
\begin{gathered}
\mathcal{F}(\boldsymbol{\Delta} \mid q)=1+\cdots+\left(p_{m n}-1\right) q^{m n}+\left(p_{m n+1}-1\right) q^{m n+1} \\
+\cdots+\left(p_{m n+N}-p_{N}\right) q^{m n+N}+\cdots
\end{gathered}
$$

This corresponds to quotienting out the module of the null state.We observe that the condition $\epsilon^{\prime}=\epsilon$, providing the character of a degenerate representation $\mathcal{V}_{m, n}$, assures that the Coulomb gas fusion condition $\alpha_{\text {int }}+\alpha_{\text {int }}=\alpha_{\text {ext }}$ is satisfied for any value of $\epsilon$.

\subsection{Characters in minimal models}

In the case of the minimal models, $b^{2}=-\frac{p^{\prime}}{p}$, where $p$ and $p^{\prime}$ are coprime positive integers, $0<p<p^{\prime}$, we know that a fully-degenerate highest-weight module $\mathcal{V}_{m, n}$ has a null-state at level $m n$, and another at level $(p-m)\left(p^{\prime}-n\right)$. We need first to solve the new poles appearing in the term $H_{m^{\prime} n^{\prime}}=\frac{R_{m^{\prime}, n^{\prime}}^{\text {tor }}(2 \epsilon)}{\left(\Delta_{m, n}^{\epsilon^{\prime}}-\Delta_{m^{\prime}, n^{\prime}}\right)}$, where $\Delta_{m, n}^{\left(\epsilon^{\prime}\right)}$ is defined in (5.3), $0<m<p$ and $0<n<p^{\prime}$, and $m^{\prime}=p-m$, and $n^{\prime}=p^{\prime}-n$.

\subsubsection{The regularization $\epsilon^{\prime \prime}$}

We introduce a third regularization parameter $\epsilon^{\prime \prime}$ to move away from the minimal model point by setting

$$
b=\sqrt{-\frac{p^{\prime}}{p}\left(1+\epsilon^{\prime \prime}\right)}
$$

For $\epsilon, \epsilon^{\prime}, \epsilon^{\prime \prime} \ll 1$,

$$
\frac{R_{m^{\prime}, n^{\prime}}^{\mathrm{tor}}(2 \epsilon)}{\left(\Delta_{m, n}^{\left(\epsilon^{\prime}\right)}-\Delta_{m^{\prime}, n^{\prime}}\right)}=-\frac{\epsilon+\mathcal{O}\left(\epsilon^{2}\right)}{-\epsilon^{\prime}+\frac{1}{2} \sqrt{-p p^{\prime}} \epsilon^{\prime \prime}+\mathcal{O}\left(\epsilon^{\prime 2}, \epsilon^{\prime \prime 2}, \epsilon^{\prime} \epsilon^{\prime \prime}\right)}
$$

Again, the final result depends on how we approach the point $\left(\epsilon, \epsilon^{\prime}, \epsilon^{\prime \prime}\right)=(0,0,0)$. In order to obtain the minimal model character we have first to remove the null state at level $(p-m)\left(p^{\prime}-n\right)$. This is obtained by setting,

$$
\epsilon^{\prime} \rightarrow \epsilon, \quad \epsilon^{\prime \prime} \rightarrow \frac{4}{\sqrt{-p p^{\prime}}} \epsilon
$$

and taking the limit $\epsilon \rightarrow 0$. Then both (5.6) and,

$$
\lim _{\epsilon \rightarrow 0} \frac{R_{m^{\prime}, n^{\prime}}^{\mathrm{tor}}(2 \epsilon)}{\left(\Delta_{m, n}^{(\epsilon)}-\Delta_{m^{\prime}, n^{\prime}}\right)}=-1
$$

are satisfied at the same time. The results (5.6) and (5.11) are not sufficient to prove that one obtains in the limit the minimal model character. One has to consider that there are 
other terms, of the form,

$$
\frac{R_{m_{1}, n_{1}}^{\mathrm{tor}}(2 \epsilon)}{\left(\Delta_{m_{1}, n_{1}}^{(\epsilon)}-\Delta_{m_{1}, n_{1}}\right)} \frac{R_{m_{2}, n_{2}}^{\mathrm{tor}}(2 \epsilon)}{\left(\Delta_{-m_{1}, n_{1}}-\Delta_{m_{2}, n_{2}}\right)},
$$

that will contribute when "resonances" in the conformal dimension, such as $\Delta_{-m_{1}, n_{1}}=$ $\Delta_{m_{2}, n_{2}}$, occur. The fact that there is an infinite number of resonances, or equivalently, infinitely-many pairs $(r, s)$ that correspond to the same conformal dimension $\Delta_{r, s}$, and that the recursion relation expression for $H_{N}$ includes all resonances $(r, s)$ such that $r s \leqslant N$ will thus play an important role. We are now in the position to give the exact contribution of all the terms which do not vanish in the limit $\epsilon \rightarrow 0$ (see appendix B for the derivation of the following results). In the following we always assume that the integers $(m, n)$ belong to the minimal model $\mathcal{M}_{p, p^{\prime}}$ Kac table, $0<m<p, 0<n<p^{\prime}$. Concerning the terms in the recursion that have at the denominator the dimension of the internal field $\Delta_{m, n}^{(\epsilon)}$, we show in appendix B that,

$$
\text { if }(m, n) \leftrightarrow(r, s)_{l}^{ \pm} \Longrightarrow \lim _{\epsilon \rightarrow 0} \frac{R_{r, s}^{\mathrm{tor}}(2 \epsilon)}{\Delta_{m, n}^{(\epsilon)}-\Delta_{r, s}}=-\frac{1}{2^{2 l-1 \pm 1}(2 l \pm 1)} \prod_{k=1}^{l-\frac{1}{2} \pm \frac{1}{2}} \frac{4 k^{2}-1}{k^{2}}
$$

We consider now the terms of the type $R_{r, s}^{\text {tor }}(2 \epsilon) /\left(\Delta_{m^{\prime},-n^{\prime}}-\Delta_{r, s}\right)$. We define two integers $\left(l_{1}, l_{2}\right)$ from the Euclidean division of $(r, s),(r, s)=\left(l_{1} p+m, l_{2} p^{\prime}+n\right)$. We have,

$$
\text { if } \begin{aligned}
&\left(m^{\prime},-n^{\prime}\right) \leftrightarrow(r, s)_{l^{\prime}}^{ \pm} \Longrightarrow \\
& \qquad \lim _{\epsilon \rightarrow 0} \frac{R_{r, s}^{\mathrm{tor}}}{\Delta_{m^{\prime},-n^{\prime}}-\Delta_{r, s}}=-\frac{1}{2^{2 l+1} l^{\prime}} \prod_{k=1}^{l} \frac{4 k^{2}-1}{k^{2}}, \quad l=\min \left(l_{1}, l_{2}\right)
\end{aligned}
$$

We will provide below the complete combinatorial structure of all the terms that contribute to the character. All these terms are finite, but they are fractional and add up to integral values.

\subsection{Example 1. The Ising model, $\left(p^{\prime}, p\right)=(4,3),(m, n)=(1,1)$}

We give here an explicit application of the previous formulas to the identity character of the $\mathcal{M}_{4,3}$ minimal model. We set $\alpha_{\text {est }}=2 \epsilon, \alpha_{\text {int }}=\epsilon$ and $b=b^{\epsilon}$ given by (5.10).

$$
\begin{aligned}
H_{1} & =\frac{R_{1,1}^{\text {tor }}(2 \epsilon)}{\left(\Delta_{1,1}^{(\epsilon)}-\Delta_{1,1}\right)}, \quad \lim _{\epsilon \rightarrow 0} H_{1}=-1, \quad H_{6}=\frac{R_{2,3}^{\text {tor }}(2 \epsilon)}{\left(\Delta_{1,1}^{(\epsilon)}-\Delta_{2,3}\right)}, \quad \lim _{\epsilon \rightarrow 0} H_{6}=-1 \\
H_{11} & =\frac{R_{1,1}^{\text {tor }}(2 \epsilon)}{\left(\Delta_{1,1}^{(\epsilon)}-\Delta_{1,1}\right)} \frac{R_{2,5}^{\text {tor }}(2 \epsilon)}{\left(\Delta_{1,-1}-\Delta_{2,5}\right)}+\frac{R_{2,3}^{\text {tor }}(2 \epsilon)}{\left(\Delta_{1,1}^{(\epsilon)}-\Delta_{2,3}\right)} \frac{R_{5,1}^{\text {tor }}(2 \epsilon)}{\left(\Delta_{2,-3}-\Delta_{5,1}\right)}
\end{aligned}
$$

Using the fact that $(1,1)$ and $(2,3)$ are in the Kac table and that $(1,-1) \rightarrow(2,5)_{1}^{-}$, $(2,-3) \rightarrow(5,1)_{1}^{+}$, one has from (5.13) and (5.14),

$$
\begin{aligned}
\lim _{\epsilon \rightarrow 0} H_{11} & =\left(-1 \times-\frac{1}{2}\right)+\left(-1 \times-\frac{1}{2}\right)=1 \\
H_{13} & =\frac{R_{1,1}^{\mathrm{tor}}(2 \epsilon)}{\left(\Delta_{1,1}^{(\epsilon)}-\Delta_{1,1}\right)} \frac{R_{4,3}^{\mathrm{tor}}(2 \epsilon)}{\left(\Delta_{-1,1}-\Delta_{4,3}\right)}+\frac{R_{2,3}^{\mathrm{tor}}(2 \epsilon)}{\left(\Delta_{1,1}^{(\epsilon)}-\Delta_{2,3}\right)} \frac{R_{1,7}^{\mathrm{tor}}(2 \epsilon)}{\left(\Delta_{2,-3}-\Delta_{1,7}\right)}
\end{aligned}
$$


From $(1,-1) \rightarrow(4,3)_{1}^{+},(2,-3) \rightarrow(1,7)_{1}^{-}$

$$
\lim _{\epsilon \rightarrow 0} H_{13}=\left(-1 \times-\frac{1}{2}\right)+\left(-1 \times-\frac{1}{2}\right)=1,
$$

These two terms correspond to adding again the null states at level 11 and 13, which are contained into the modules of both the null states at level 1 and 6 , and were therefore subtracted twice. Let us make another example at level 20,

$$
\begin{aligned}
H_{20}= & \frac{R_{1,1}^{\text {tor }}(2 \epsilon)}{\left(\Delta_{1,1}^{(\epsilon)}-\Delta_{1,1}\right)} \frac{R_{4,3}^{\text {tor }}(2 \epsilon)}{\left(\Delta_{1,-1}-\Delta_{4,3}\right)} \frac{R_{7,1}^{\text {tor }}(2 \epsilon)}{\left(\Delta_{4,-3}-\Delta_{7,1}\right)} \\
& +\frac{R_{1,1}^{\text {tor }}(2 \epsilon)}{\left(\Delta_{1,1}^{(\epsilon)}-\Delta_{1,1}\right)} \frac{R_{2,5}^{\text {tor }}(2 \epsilon)}{\left(\Delta_{1,-1}-\Delta_{2,5}\right)} \frac{R_{1,9}^{\text {tor }}(2 \epsilon)}{\left(\Delta_{2,-5}-\Delta_{1,9}\right)} \\
& +\frac{R_{2,3}^{\text {tor }}(2 \epsilon)}{\left(\Delta_{1,1}^{(\epsilon)}-\Delta_{2,3}\right)} \frac{R_{5,1}^{\text {tor }}(2 \epsilon)}{\left(\Delta_{2,-3}-\Delta_{5,1}\right)} \frac{R_{1,9}^{\text {tor }}(2 \epsilon)}{\left(\Delta_{5,-1}-\Delta_{1,9}\right)} \\
& +\frac{R_{2,3}^{\text {tor }}(2 \epsilon)}{\left(\Delta_{1,1}^{(\epsilon)}-\Delta_{2,3}\right)} \frac{R_{1,7}^{\text {tor }}(2 \epsilon)}{\left(\Delta_{2,-3}-\Delta_{1,7}\right)} \frac{R_{7,1}^{\text {tor }}(2 \epsilon)}{\left(\Delta_{1,-7}-\Delta_{7,1}\right)}+\frac{R_{4,5}^{\text {tor }}(2 \epsilon)}{\left(\Delta_{1,1}^{(\epsilon)}-\Delta_{4,5}\right)}
\end{aligned}
$$

From (5.13) and (5.14),

$$
\begin{aligned}
\lim _{\epsilon \rightarrow 0} H_{20}= & \left(-1 \times-\frac{1}{2} \times-\frac{1}{2}\right)+\left(-1 \times-\frac{1}{2} \times-\frac{1}{2}\right) \\
& +\left(-1 \times-\frac{1}{2} \times-\frac{1}{4}\right)+\left(-1 \times-\frac{1}{2} \times-\frac{1}{4}\right)-\frac{1}{4}=-1
\end{aligned}
$$

Notice that this sum of terms which add up to an integer has exactly the same structure as equation (3.18).

\subsection{General case}

We consider the character of a representation indexed by $(m, n), 0<m<p, 0<n<p^{\prime}$. We provide here the explicit procedure to find all the terms that, in the limit (5.10), have a finite fraction contribution that sums up, at a given level, to 1 or -1 . We want to give a procedure that takes into account all the singular terms appearing in the one-point torus recursion relation in the minimal model limit. Given a pair of indices $(m, n)$, we want to find the set of pairs $(r, s)$, that are resonant with $(m,-n),(m,-n) \leftrightarrow(r, s)_{l}^{+}$, or with $(-m, n),(-m, n) \leftrightarrow(r, s)_{l}^{+}$, where we used the notation defined in (2.13). These pairs are obtained respectively by the two transformations,

$$
\begin{array}{lll}
v_{1}^{(l)}: & (m, n) \rightarrow\left(r=l p+m, s=l p^{\prime}-n\right) & (m,-n) \leftrightarrow(r, s)_{l}^{+} \\
v_{2}^{(l)}: & (m, n) \rightarrow\left(r=l p-m, s=l p^{\prime}+n\right) & (-m, n) \leftrightarrow(r, s)_{l}^{+}
\end{array}
$$


By using these transformations we generate the following diagram,

$$
k=0
$$

$k=1$
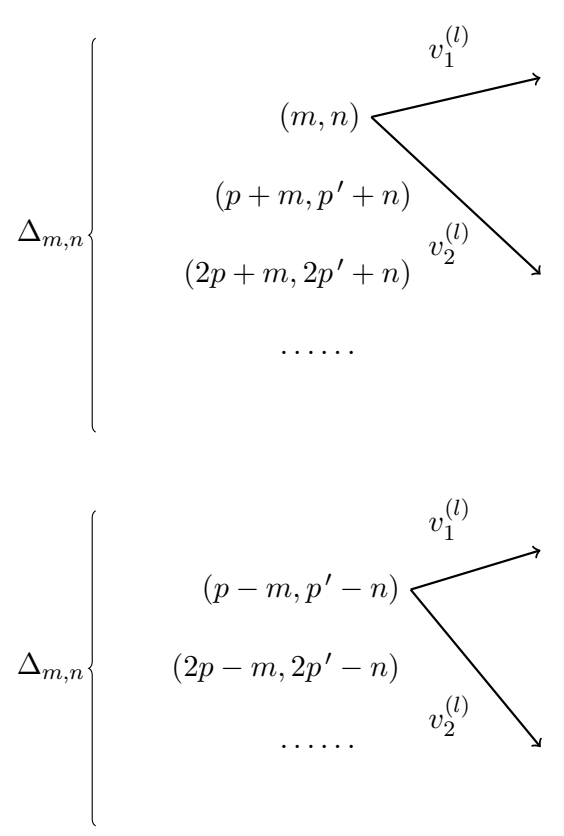

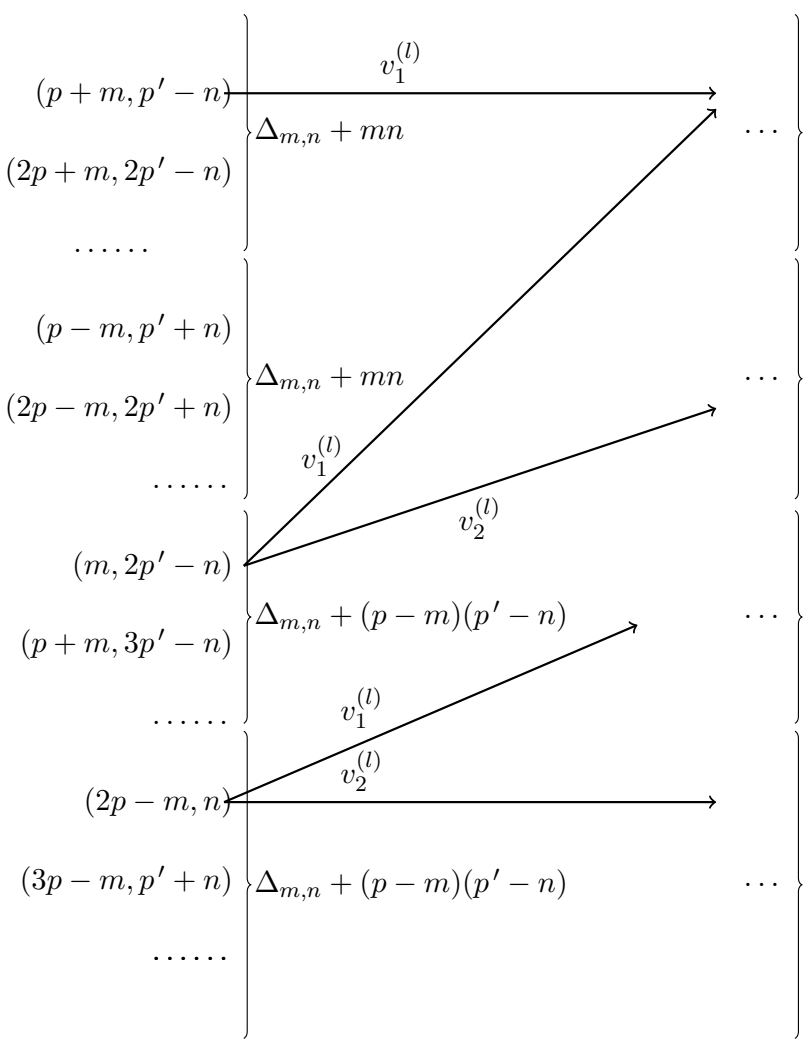

In the $k=0$ column, we place the two groups of pairs, $(r, s)$, which are resonant with $(m, n),(m, n) \leftrightarrow(r, s)_{l}^{+}$and $(m, n) \leftrightarrow(r, s)_{l}^{-}$. The column $k=1$ is generated by applying the transformations $v_{1}^{(l)}$ and $v_{2}^{(l)}$ to $(m, n)$ and $\left(p^{\prime}-m, p-n\right)$. One obtains four families, corresponding to the two set of pairs $(r, s)$ resonant with $(m,-n),(m,-n) \leftrightarrow(r, s)_{l}^{+}$ and $(m,-n) \leftrightarrow(r, s)_{l}^{-}$and associated to representation with dimension $\Delta_{m, n}+m n$, plus the two sets of pairs in resonance with $\left(p-m, n-p^{\prime}\right),\left(p-m, n-p^{\prime}\right) \leftrightarrow(r, s)_{l}^{+}$and $\left(p-m, n-p^{\prime}\right) \leftrightarrow(r, s)_{l}^{-}$and associated to representation with dimension $\Delta_{m, n}+\left(p^{\prime}-\right.$ $m)(p-n)$. The column $k=2$ is obtained by applying the transformations $v_{1}^{(l)}$ and $v_{2}^{(l)}$ to $\left(p+m, p^{\prime}-n\right),\left(p-m, p^{\prime}+n\right),\left(m, 2 p^{\prime}-n\right)$ and $(2 p-m, n)$ and so on. At each column one can therefore identify four families of pairs that we indicate with the letters $U^{1}, D^{1}$ and $U^{2}, D^{2}$. Any pair of indices appearing in the diagram is identified by its family and by two non-negative integers $k$ and $l$, indicating respectively the column and the position in the interior of each family,

$$
\begin{aligned}
& U^{1}(k, l) \rightarrow\left((k+l) p+m,(-1)^{k} n+l p^{\prime}+\frac{1-(-1)^{k}}{2} p^{\prime}\right) \\
& D^{1}(k, l) \rightarrow\left((1+l) p-m,(-1)^{k+1} n+(l+k) p^{\prime}+\frac{1-(-1)^{k+1}}{2} p^{\prime}\right)
\end{aligned}
$$




$$
\begin{aligned}
& U^{2}(k, l) \rightarrow\left(l p+m,(-1)^{k} n+(l+k) p^{\prime}+\frac{1-(-1)^{k}}{2} p^{\prime}\right) \\
& D^{2}(k, l) \rightarrow\left((1+l+k) p-m,(-1)^{k+1} n+l p^{\prime}+\frac{1-(-1)^{k+1}}{2} p^{\prime}\right)
\end{aligned}
$$

It is straightforward to obtain the action of the transformations $v_{1}^{(l)}$ and $v_{2}^{(l)}$ on each index,

$$
\begin{array}{ll}
v_{1}^{\left(l^{\prime}\right)}: U^{1}(k, l) \rightarrow U^{1}\left(k+2 l+1, l^{\prime}\right), & U^{2}(k, l) \rightarrow U^{1}\left(k+2 l+1, l^{\prime}\right) \\
v_{1}^{\left(l^{\prime}\right)}: D^{1}(k, l) \rightarrow D^{2}\left(k+2 l+1, l^{\prime}\right), & D^{2}(k, l) \rightarrow D^{2}\left(k+2 l+1, l^{\prime}\right) \\
v_{2}^{\left(l^{\prime}\right)}: U^{1}(k, l) \rightarrow D^{1}\left(k+2 l+1, l^{\prime}\right), & U^{2}(k, l) \rightarrow D^{1}\left(k+2 l+1, l^{\prime}\right) \\
v_{2}^{\left(l^{\prime}\right)}: D^{1}(k, l) \rightarrow U^{2}\left(k+2 l+1, l^{\prime}\right), & D^{2}(k, l) \rightarrow U^{2}\left(k+2 l+1, l^{\prime}\right) .
\end{array}
$$

The above rules allow to write the chains of resonant terms in the recursion relation in terms of words formed by the letters $U^{1,2}$ and $D^{1,2}$, whose sequences have to satisfy the connections above. For instance, the terms in the example in (5.19), correspond to the following words,

$$
\begin{aligned}
& U^{1}(0,0) U^{1}(1,0) U^{1}(2,0) \\
& U^{1}(0,0) D^{1}(1,0) U^{2}(2,0) \\
& D^{1}(0,0) D^{2}(1,0) U^{2}(2,0) \\
& D^{1}(0,0) U^{2}(1,0) U^{1}(2,0) \\
& U^{1}(0,1)
\end{aligned}
$$

As seen in this example, the words corresponding to a certain level $N$ will all end either with $U$ or with $D$. The reason is that the last arrows of the chains must point to the same dimension, so they must all point either to the 1 sector or all point to the 2 sector. From (5.24), all $U$ labels transform to a label in the 1 sector, while all $D$ labels transform to labels in the 2 sector. Since there always exists either a $U^{1}(0,0) U^{1}(1,0) \cdots U^{1}(K, 0)$ or a $D^{2}(0,0) D^{2}(1,0) \cdots D^{2}(K, 0)$ chain, of length $K+1$, the level $N$ is either,

$$
N_{U}(K)=\sum_{k=0}^{K}(k p+m)\left((-1)^{k} n+\left(1-(-1)^{k}\right) \frac{p^{\prime}}{2}\right),
$$

or

$$
N_{D}(K)=\sum_{k=0}^{K}((k+1) p-m)\left((-1)^{k+1} n+\left(1-(-1)^{k+1}\right) \frac{p^{\prime}}{2}\right)
$$

Then, the contribution of each word is obtained by using formulas (5.13) and (5.14). For instance,

$$
\begin{aligned}
& U^{1}\left(k_{1}, l_{1}\right) U^{1}\left(k_{1}+2 l_{1}+1, l_{2}\right) \rightarrow \lim _{\epsilon \rightarrow 0} \frac{R_{r_{2}, s_{2}}^{\text {tor }}(2 \epsilon)}{\Delta_{r_{1},-s_{1}}-\Delta_{r_{2}, s_{2}}} \\
& \text { with } \quad\left(r_{1}, s_{1}\right)=U^{1}\left(k_{1}, l_{1}\right), \quad\left(r_{2}, s_{2}\right)=U^{1}\left(k_{1}+2 l_{1}+1, l_{2}\right)
\end{aligned}
$$


Applying (5.14) with $l^{\prime}=l_{1}+l_{2}+1$ and $l=l_{2}$, one has,

$$
U^{1}\left(k_{1}, l_{1}\right) U^{1}\left(k_{1}+2 l_{1}+1, l_{2}\right) \rightarrow-\frac{1}{2^{2 l_{2}+1}\left(l_{1}+l_{2}+1\right)} \prod_{j=1}^{l_{2}} \frac{4 \lambda^{2}-1}{\lambda^{2}}
$$

In the same way, we find,

$$
\begin{aligned}
U^{2}\left(k_{1}, l_{1}\right) D^{1}\left(k_{1}+2 l_{1}+1, l_{2}\right) & =D^{1}\left(k_{1}, l_{1}\right) U^{2}\left(k_{1}+2 l_{1}+1, l_{2}\right) \\
& =D^{2}\left(k_{1}, l_{1}\right) D^{2}\left(k_{1}+2 l_{1}+1, l_{2}\right) \\
& =U^{1}\left(k_{1}, l_{1}\right) U^{1}\left(k_{1}+2 l_{1}+1\right) \\
& =-\frac{1}{2^{2 l_{2}+1}\left(l_{1}+l_{2}+1\right)} \prod_{\lambda=1}^{l_{2}} \frac{4 \lambda^{2}-1}{\lambda^{2}}
\end{aligned}
$$

and

$$
\begin{aligned}
U^{2}\left(k_{1}, l_{1}\right) U^{1}\left(k_{1}+2 l_{1}+1\right) & =D^{1}\left(k_{1}, l_{1}\right) D^{2}\left(k_{1}+2 l_{1}+1, l_{2}\right) \\
& =D^{2}\left(k_{1}, l_{1}\right) U^{2}\left(k_{1}+2 l_{1}+1, l_{2}\right) \\
& =U^{1}\left(k_{1}, l_{1}\right) D^{1}\left(k_{1}+2 l_{1}+1, l_{2}\right) \\
& =-\frac{1}{2^{2 l_{2}+1}\left(k_{1}+l_{1}+l_{2}+1\right)} \prod_{\lambda=1}^{l_{2}} \frac{4 \lambda^{2}-1}{\lambda^{2}}
\end{aligned}
$$

The non-trivial contribution at level $N(K)$ is given by all possible chains starting with $U^{1}\left(0, l_{0}\right)$ or $D^{1}\left(0, l_{0}\right)$, with constraint on the last terms: $U^{1}$ or $U^{2}$ if $N=N_{1}, D^{1}$ or $D^{2}$ if $N=N_{2}$. Note that given equalities (5.29) and (5.30), for fixed $K$ and fixed $\left\{l_{i}\right\}$, the contributions at levels $N_{1}(K)$ and $N_{2}(K)$ are equal. Therefore, to compute the contribution at level $N_{1}(K)$ (resp. at level $N_{2}(K)$ ), instead of constraining the chains to end by $U$ (resp. $D)$, we can leave the ends of the chains free and divide by 2 at the end. The first terms of the chains involve the internal dimension $\Delta_{m, n}^{\epsilon}$,

$$
\begin{aligned}
& U^{1}\left(0, l_{0}\right)=\frac{R_{l_{0} p+m, l_{0} p^{\prime}+n}^{\mathrm{tor}}}{\Delta_{m, n}^{\epsilon}-\Delta_{l_{0} p+m, l_{0} p^{\prime}+n}}=-\frac{1}{2^{2 l_{0}}\left(2 l_{0}+1\right)} \prod_{\lambda=1}^{l_{0}} \frac{4 \lambda^{2}-1}{\lambda^{2}} \\
& D^{1}\left(0, l_{0}\right)=\frac{R_{\left(l_{0}+1\right) p-m,\left(l_{0}+1\right) p^{\prime}-n}^{\mathrm{tor}}}{\Delta_{m, n}^{\epsilon}-\Delta_{\left(l_{0}+1\right) p-m,\left(l_{0}+1\right) p^{\prime}-n}}=-\frac{1}{2^{2 l_{0}}\left(2 l_{0}+1\right)} \prod_{\lambda=1}^{l_{0}} \frac{4 \lambda^{2}-1}{\lambda^{2}}
\end{aligned}
$$

We now need to specify the set $\left\{l_{i}\right\}$. Let us denote $I$ the cardinal of this set. We then have the constraint,

$$
I+\sum_{i=0}^{I-1} 2 l_{i}=K+1
$$

By writing $I=K+1-2 a$, the constraint is,

$$
\sum_{i=0}^{K-2 a} l_{i}=a
$$


and the set $\left\{l_{0}, l_{1}, \cdots, l_{K-2 a}\right\}$ is therefore a partition of size $K-2 a+1$ of the integer $a$, $\left\{l_{0}, l_{1}, \cdots, l_{K-2 a}\right\}=\tilde{p}_{K-2 a+1}(a)$ in which zeroes are included, as well as all permutations. For example the set of the partitions of size 3 of 3 is,

$$
\{(3,0,0),(0,3,0),(2,1,0),(2,0,1),(1,2,0),(1,0,2),(0,1,2),(0,2,1),(1,1,1)\} .
$$

$a$ then runs from 0 to a maximal $a$. If $\mathrm{K}$ is odd, $K+1$ is even and $I_{\min }=K+1-2 a_{\max }$ is even. Therefore $I_{\min }=2$, and $a_{\max }=(K-1) / 2$. If $K$ is even, $K+1$ is odd and $I_{\min }=K+1-2 a_{\max }$ is odd and equals 1 . Therefore $a_{\max }=K / 2$ and $l_{0}=a_{\max }$, all other $l_{i}$ being 0 , and this chain consists of the single term (5.31). We can now give the final formula for the contribution $C_{N(K)}$ that takes into account all the non-zero terms at a given level $N(K)$,

$$
\begin{aligned}
C_{N(K)}= & \frac{1}{2} \sum_{a=0}^{\left[\frac{K-1}{2}\right]} \sum_{\left\{l_{0}, \cdots, l_{K-2 a}\right\}=\tilde{p}_{K-2 a+1}(a)} \sum_{\left\{X_{0}, \cdots, X_{K-2 a}\right\}} X_{0}\left(0, l_{0}\right) \\
& \times \prod_{j=0}^{K-1-2 a} X_{j}\left(2 \sum_{i=0}^{j-1} l_{i}+j, l_{j}\right) X_{j+1}\left(2 \sum_{i=0}^{j} l_{i}+j+1, l_{j+1}\right) \\
& +\delta_{K(\bmod 2), 0} X_{0}(0, K / 2),
\end{aligned}
$$

where the $X_{j>1}$ 's are $U^{1,2}$ or $D^{1,2}, X_{0}$ is $U^{1}$ or $D^{1}$. From (5.29), (5.30) and (5.31),

$$
\begin{aligned}
C_{N(K)}= & -\delta_{K(\bmod 2), 0} \frac{\prod_{\lambda=1}^{K / 2} \frac{4 \lambda^{2}-1}{\lambda^{K}}(K+1)}{2^{K}\left(\frac{K-1}{2}\right]} \sum_{a=0}^{\left[l_{0}, \ldots, l_{K-2 a}\right\}=\tilde{p}_{K-2 a+1}(a)}-\frac{4 \lambda^{2}-1}{2^{2 l_{0}\left(2 l_{0}+1\right)}} \\
& \times \prod_{j=0}^{l_{0}}-\frac{1}{l^{2} l_{j+1}+1}\left(\frac{1}{l_{j}+l_{j+1}+1}+\frac{1}{l_{j}+l_{j+1}+1+2 \sum_{i=0}^{j-1} l_{i}+j}\right) .
\end{aligned}
$$

We have checked numerically - up to order $N \sim 300-$ that $C_{N(K)}=(-1)^{K+1}$.

\section{Conclusions}

We extended Zamolodchikov's elliptic recursion relation for 4-point conformal blocks on the sphere [15], and its analogue for 1-point functions on the torus [3, 10], originally derived for conformal blocks in Liouville theory with non-rational central charge, to conformal field theories with rational central charges, including the generalized minimal and minimal models. When the central charge is rational, solutions of the recursion relation have additional poles that appear on a term by term basis. These poles are non physical in the sense that they are artifacts of the recursion which splits perfectly well-defined terms into terms that can be singular on their own but add up to finite contributions. We studied the structure 
of these non physical poles in two situations. 1. In 4-point conformal blocks on the sphere, where we found that the singular terms add up to finite terms on the basis of examples, and conjectured that this is the case in general, and that regularizing properly all the parameters entering the conformal block, one obtains the minimal model conformal block. 2 . In 1-point conformal blocks on the torus, in the limit where the vertex operator insertion is the identity operator and the 1-point conformal block reduces to a 0-point conformal block, which is a Virasoro character. In this case, the contributions of the non-physical poles are fractions, and explicit expressions of these fractions were derived in (5.13) and (5.14). We unveiled the combinatorial structure of these fractions found it to be reminiscent of that in the Feigin-Fuks construction of minimal model characters [4], and used it to show that the contribution of the non-physical poles add up to \pm 1 . The non-physical poles of the 4-point conformal blocks also follow this combinatorial structure. A fine regularization of the central charge is needed in the case of the 0-point functions, whereas the 4-point function is not sensitive to the regularization used.

\section{Acknowledgments}

We thank Kenji Iohara, Santiago Migliaccio, Rubik Poghossian and Sylvain Ribault for discussions. OF wishes to thank Profs K Lechner, M Matone and D Sorokin for hospitality in the Physics Department, University of Padova, while this work was in progress, and the organizers of "Supersymmetric Quantum Field Theories in the Non-perturbative Regime", and Prof A Dabholkar for hospitality at the Galileo Galilei Institute for Theoretical Physics, Arcetri, Firenze, Italy, and at the Abdus Salam Center for Theoretical Physics, Trieste, respectively, where it was finalized. OF is supported by a Special Studies Program grant from the Faculty of Science, University of Melbourne, and the Australian Research Council.

\section{A A direct computation at $c=-2$}

At $c=-2$, which we can consider as the $\mathcal{M}(2,1)$ minimal model, the first extra pole appear at order 3 in the expansion of the conformal block. It is therefore possible to compare the recursion result to the result one gets by hand, ie by computing the Shapovalov matrix of inner products and the "matrix elements",

$$
\begin{array}{r}
\left\langle L_{-Y} \Delta \mid \Phi_{\Delta_{1}}(x) \Phi_{\Delta_{2}}(0)\right\rangle /\left\langle\Delta \mid \Phi_{\Delta_{1}}(x) \Phi_{\Delta_{2}}(0)\right\rangle, \\
\left\langle\Phi_{\Delta_{3}}(1) \Phi_{\Delta_{4}}(\infty) \mid L_{-Y^{\prime}} \Delta\right\rangle /\left\langle\Phi_{\Delta_{3}}(1) \Phi_{\Delta_{4}}(\infty) \mid \Delta\right\rangle
\end{array}
$$

which appear in (3.3). In the basis $\left\{L_{-1}^{3}|\Delta\rangle, L_{-1}\left|Q_{2}\right\rangle,\left|Q_{3}\right\rangle\right\}$ where,

$$
\begin{aligned}
& \left|Q_{2}\right\rangle=\left(L_{-1}^{2}-\frac{2(2 \Delta+1)}{3} L_{-2}\right)|\Delta\rangle \\
& \left|Q_{3}\right\rangle=\left(L_{-1}^{3}-2(\Delta+1) L_{-2} L_{-1}+\Delta(\Delta+1) L_{-3}\right)|\Delta\rangle
\end{aligned}
$$


are the quasi-primary states at levels 2 and 3, the Shapovalov matrix at level 3 is diagonal,

$$
\begin{aligned}
S_{(3)}=\operatorname{diag}(24 \Delta & (\Delta+1)(2 \Delta+1), \\
& \frac{64}{9}(\Delta+2)(2 \Delta+1)\left(\Delta-\Delta_{1,2}\right)\left(\Delta-\Delta_{2,1}\right), \\
& \left.6 \Delta(\Delta+1)(\Delta+2)\left(\Delta-\Delta_{1,3}\right)\left(\Delta-\Delta_{3,1}\right)\right)
\end{aligned}
$$

Then the contribution of the quasi-primary at level 3 is,

$$
\frac{P_{L}\left(Q_{3} ; \Delta_{1}, \Delta_{2}\right) P_{R}\left(Q_{3} ; \Delta_{3}, \Delta_{4}\right)}{\left\langle Q_{3} \mid Q_{3}\right\rangle}=\frac{\mathcal{P}_{2}\left(\Delta ; \Delta_{i}\right)}{6 \Delta(\Delta+1)(\Delta+2)\left(\Delta-\Delta_{1,3}\right)\left(\Delta-\Delta_{3,1}\right)}
$$

where $\mathcal{P}_{2}\left(\Delta ; \Delta_{i}\right)$ is a polynomial of order 2 in the internal dimension $\Delta$ :

$$
\begin{aligned}
\mathcal{P}_{2}\left(\Delta ; \Delta_{i}\right)= & \left(\Delta_{1}-\Delta_{2}\right)\left(\Delta_{3}-\Delta_{4}\right)\left(\Delta_{1}+\Delta_{2}-1\right)\left(\Delta_{3}+\Delta_{4}-1\right) \Delta^{2} \\
& +\left(\Delta_{1}-\Delta_{2}\right)\left(\Delta_{3}-\Delta_{4}\right)\left\{\left(1-\left(\Delta_{1}+\Delta_{2}\right)\right)\left(\left(\Delta_{3}-\Delta_{4}\right)^{2}-\left(\Delta_{3}+\Delta_{4}\right)\right)\right. \\
& \left.+\left(1-\left(\Delta_{3}+\Delta_{4}\right)\right)\left(\left(\Delta_{1}-\Delta_{2}\right)^{2}-\left(\Delta_{1}+\Delta_{2}\right)\right)\right\} \Delta \\
& +\left(\Delta_{1}-\Delta_{2}\right)\left(\Delta_{3}-\Delta_{4}\right)\left(\left(\Delta_{1}-\Delta_{2}\right)^{2}-\left(\Delta_{1}+\Delta_{2}\right)\right) \\
& \times\left(\left(\Delta_{3}-\Delta_{4}\right)^{2}-\left(\Delta_{3}+\Delta_{4}\right)\right)
\end{aligned}
$$

When $c=-2$, we have,

$$
\Delta_{1,3}=0, \quad \Delta_{3,1}=3,
$$

and the contribution (A.4) of $\left|Q_{3}\right\rangle$ is well-defined. However, if we decompose it in partial fractions,

$$
\frac{\left\langle Q_{3}\left|\Phi_{\Delta_{1}}(0)\right| \Delta_{2}\right\rangle\left\langle\Delta_{3}\left|\Phi_{\Delta_{4}}(\infty)\right| Q_{3}\right\rangle}{\left\langle Q_{3} \mid Q_{3}\right\rangle}=\frac{A}{\Delta-\Delta_{1,1}}+\frac{B}{\Delta-\Delta_{1,3}}+\frac{C}{\Delta-\Delta_{3,1}}+\frac{A \Delta+D}{(\Delta+1)(\Delta+2)},
$$

we find,

$$
\begin{aligned}
A & =\frac{f_{1,1}\left(\Delta_{1}, \Delta_{2}\right) f_{1,1}\left(\Delta_{3}, \Delta_{4}\right)}{2 \Delta_{1,3} \Delta_{3,1}}, \\
B & =\frac{f_{1,3}\left(\Delta_{1}, \Delta_{2}\right) f_{1,3}\left(\Delta_{3}, \Delta_{4}\right)}{\Delta_{1,3}\left(\Delta_{1,3}-\Delta_{3,1}\right)\left(1+\Delta_{1,3}\right)\left(2+\Delta_{1,3}\right)}, \\
C & =\frac{f_{3,1}\left(\Delta_{1}, \Delta_{2}\right) f_{3,1}\left(\Delta_{3}, \Delta_{4}\right)}{\Delta_{3,1}\left(\Delta_{1,3}-\Delta_{3,1}\right)\left(1+\Delta_{3,1}\right)\left(2+\Delta_{3,1}\right)},
\end{aligned}
$$

where we defined the function,

$$
f_{r, s}\left(\Delta_{i}, \Delta_{j}\right)=\left(\Delta_{i}-\Delta_{j}\right)\left(\left(\Delta_{i}-\Delta_{j}\right)^{2}-\left(\Delta_{i}+\Delta_{j}\right)\left(1+\Delta_{r, s}\right)+\Delta_{r, s}\right)
$$

Notice that given equations (A.6), $A$ and $B$ become singular for $c=-2$. In fact we have,

$$
\lim _{c \rightarrow-2} A \propto \frac{R_{1,1}\left(\Delta_{i}\right) R_{2,1}\left(\Delta_{i}\right)}{\Delta_{1,-1}-\Delta_{2,1}}, \quad \lim _{c \rightarrow-2} B \propto R_{1,3}\left(\Delta_{i}\right), \quad \lim _{c \rightarrow-2} C \propto R_{3,1}\left(\Delta_{i}\right)
$$

$A$ and $B$ are the terms that add to a finite contribution. In that sense, the extra poles are artifacts of the recursion relation rewrites a well-defined quantity as a sum of terms that are individually singular. 


\section{B About $\boldsymbol{R}_{m, n}^{\text {tor }}$}

We prove that

$$
R_{m, n}^{\mathrm{tor}}(2 \epsilon)=-\epsilon\left(m b+n b^{-1}\right)+\mathcal{O}\left(\epsilon^{2}\right),
$$

for general $b$ and for all $(m, n)$.

$$
\begin{aligned}
R_{m, n}^{\mathrm{tor}}(\alpha) & =\frac{1}{4 r_{m, n}} \prod_{k, l}\left(\frac{1-k}{2} b+\frac{1-l}{2} b^{-1}-\alpha\right) \\
k & =1-2 m, 3-2 m, \cdots, 2 m-1, \quad l=1-2 n, 3-2 n, \cdots, 2 n-1
\end{aligned}
$$

and,

$$
\begin{aligned}
r_{m, n} & =-\frac{1}{2} \prod_{\rho, \sigma} 2 \lambda_{\rho, \sigma}, \\
\rho & =1-m, 2-m, \cdots, m, \quad \sigma=1-n, 2-n, \cdots, n, \quad(\rho, \sigma) \neq(0,0),(m, n)
\end{aligned}
$$

which can be rewritten as,

$$
r_{m, n}=\frac{1}{2} \prod_{i=1-m}^{m} \prod_{j=1-n}^{n}\left(i b+j b^{-1}\right), \quad(i, j) \neq(0,0), \quad(i, j) \neq(m, n),
$$

When the inserted operator is the identity, $\alpha=2 \epsilon$, we get,

$$
R_{m, n}^{\mathrm{tor}}=-\frac{\epsilon}{\prod_{i j}\left(i b+j b^{\prime}\right)} \prod_{(k, l) \neq(1,1)}\left(\frac{1-k}{2} b+\frac{1-l}{2} b^{-1}\right)+\mathcal{O}\left(\epsilon^{2}\right)
$$

We can call $\rho=\frac{1-k}{2}$ and $\sigma=\frac{1-l}{2}$, then $\rho$ goes from $1-m, 2-m, \cdots, m$ and $\sigma$ from $1-n, 2-n, \cdots, n . \quad(k, l) \neq(1,1) \Leftrightarrow(\rho, \sigma) \neq(0,0)$ and we get,

$$
\begin{aligned}
R_{m, n}^{\mathrm{tor}} & =-\epsilon\left(\prod_{(i, j) \neq(0,0),(i, j) \neq(m, n)}\left(i b+\frac{j}{b}\right)\right)^{-1}\left(\prod_{(\rho, \sigma) \neq(0,0)}\left(\rho b+\frac{\sigma}{b}\right)+\mathcal{O}\left(\epsilon^{2}\right)\right) \\
& =-\epsilon\left(m b+n b^{\prime}\right)+\mathcal{O}\left(\epsilon^{2}\right)
\end{aligned}
$$

This also implies that,

$$
\lim _{\epsilon \rightarrow 0} \frac{R_{m, n}(2 \epsilon)}{\epsilon\left(m b+n b^{-1}\right)}=-1
$$

for all $b$, for all $(m, n)$. When $c \in \mathbb{Q}$, it can happen that the denominator $r_{m, n}$ vanishes. We will show that the coefficients $R_{m, n}(2 \epsilon)$ are always well-defined and express them in closed form. Let's first examine the space $(m, n)$ for which $r_{m, n} \sim \epsilon^{d}$. Let us start with the case $d=1 . r_{m, n}=\epsilon \Rightarrow \exists\left(i_{1}, j_{1}\right)$ such that,

$$
b^{2}=-\frac{j_{1}}{i_{1}}, \quad 1-m \leqslant i_{1} \leqslant m, \quad 1-n \leqslant j_{1} \leqslant n, \quad\left(i_{1}, j_{1}\right) \neq(m, n),
$$

so that,

$$
\left.r_{m, n}=\epsilon, \quad(m, n) \in\{p\} \times\right] p^{\prime}, \infty[\bigcup] p, \infty\left[\times\left\{p^{\prime}\right\}\right.
$$




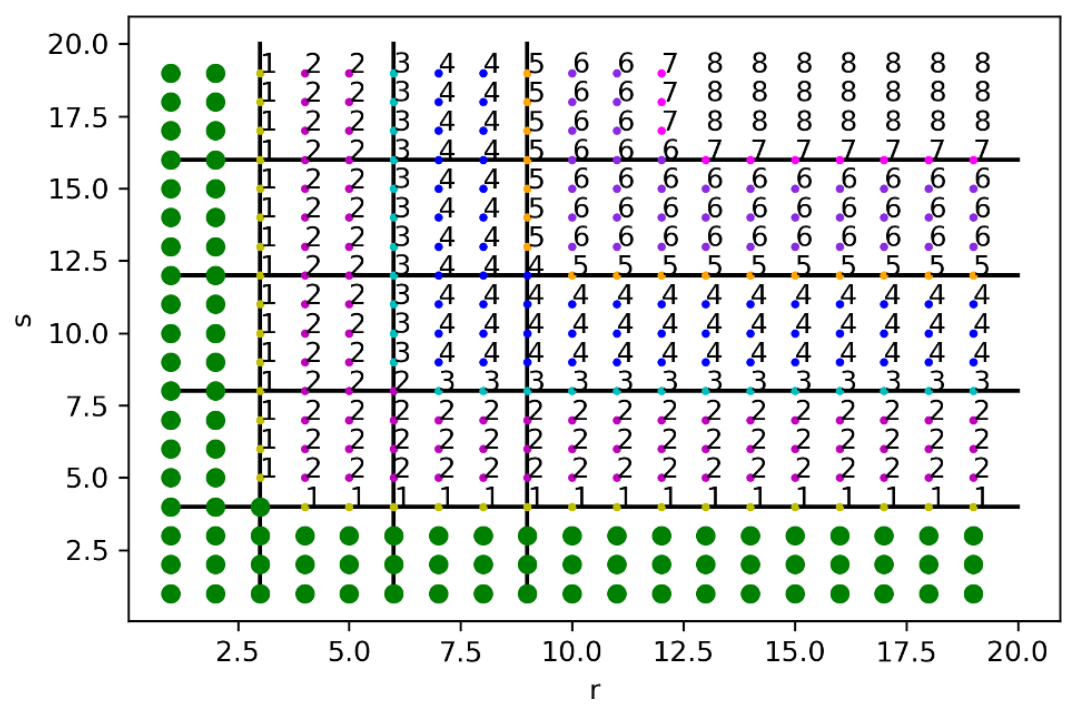

Figure 1. Degree $d$ for the Ising model $p=4, p^{\prime}=3$. Green dots are the values for which $D_{m, n} \neq 0$.

In the same way, $r_{m, n}=\epsilon^{2} \Rightarrow \exists\left(i_{1}, j_{1}\right),\left(i_{2}, j_{2}\right)$ such that $b^{2}=-\frac{j_{1}}{i_{1}}=-\frac{j_{2}}{i_{2}}$ with $\left(i_{1}, j_{1}\right) \neq$ $\left(i_{2}, j_{2}\right)$, that is, $\left(i_{1}, j_{1}\right)=\left(p, p^{\prime}\right)$ and $\left(i_{2}, j_{2}\right)=\left(-p,-p^{\prime}\right)$, that is,

$$
\left.r_{m, n}=\epsilon^{2}, \quad(m, n) \in\right] p, \infty[\times] p^{\prime}, 2 p^{\prime}[\bigcup] p, 2 p[\times] p^{\prime}, \infty[
$$

We can generalize to $r_{m, n}=\epsilon^{d}$.

$$
\begin{array}{ll}
\left.(m, n) \in\left\{\frac{d+1}{2} p\right\} \times\right] \frac{d+1}{2} p^{\prime}, \infty[\bigcup] \frac{d+1}{2} p, \infty\left[\times\left\{\frac{d+1}{2} p^{\prime}\right\}\right. & d \text { odd } \\
(m, n) \in] \frac{d}{2} p, \infty[\times] \frac{d}{2} p^{\prime},\left(\frac{d}{2}+1\right) p^{\prime}[\bigcup] \frac{d}{2} p,\left(\frac{d}{2}+1\right) p[\times] \frac{d}{2} p^{\prime}, \infty[\quad d \text { even }
\end{array}
$$

This space is shown on figure 1 for the Ising model. Notice that odd $d$ corresponds to the borders of the cells (the fundamental cell being the Kac table). The physical states are not located on the borders, so under the condition that $R_{m, n}^{\text {tor }}(2 \epsilon)$ vanishes for odd $d$, we can restrict only to even $d$. For even $d$, the coefficient $R_{m, n}^{\text {tor }}$ is,

$$
\begin{aligned}
R_{m, n}^{\mathrm{tor}}(2 \epsilon)= & -2 \epsilon\left(m \sqrt{-\frac{p^{\prime}}{p}}+\frac{n}{\sqrt{-\frac{p^{\prime}}{p}}}+\mathcal{O}(\epsilon)\right) \times \prod_{k=1}^{\frac{d}{2}} b^{-1}\left( \pm k p b^{2} \pm k p^{\prime}-2 \epsilon b\right) \\
& \times \prod^{\prime}\left(\rho b+\sigma b^{-1}-2 \epsilon\right) \times \frac{1}{2 \prod_{k=1}^{\frac{d}{2}} b^{-1}\left( \pm k p b^{2} \pm k p^{\prime}\right) \times \prod^{\prime}\left(\rho b+\sigma b^{-1}\right)}
\end{aligned}
$$

where $\prod^{\prime}$ is $\prod_{\rho=1-m}^{m} \prod_{\sigma=1-n}^{n}$ with $(0,0),(m, n)$ and $\left( \pm k p, \pm k p^{\prime}\right)$ excluded. Using the regularization for the $b$,

$$
b=\sqrt{-\frac{p^{\prime}}{p}\left(1+\frac{4 \epsilon}{\sqrt{-p p^{\prime}}}\right)}
$$


we have,

$$
\begin{aligned}
\pm k p b^{2} \pm k p^{\prime} & = \pm 4 \epsilon k \sqrt{-\frac{p^{\prime}}{p}}+\mathcal{O}\left(\epsilon^{2}\right), \\
\pm k p b^{2} \pm k p^{\prime}-2 \epsilon b & =2 \epsilon \sqrt{-\frac{p^{\prime}}{p}}( \pm 2 k-1)+\mathcal{O}\left(\epsilon^{2}\right)
\end{aligned}
$$

which gives,

$$
R_{m, n}^{\mathrm{tor}}(2 \epsilon)=-\epsilon\left(m \sqrt{-\frac{p^{\prime}}{p}}+\frac{n}{\sqrt{-\frac{p^{\prime}}{p}}}\right) \times \frac{1}{2^{d}} \prod_{k=1}^{\frac{d}{2}} \frac{4 k^{2}-1}{k^{2}}+\mathcal{O}\left(\epsilon^{2}\right)
$$

for all $(m, n)$ and $d$. For given $(m, n)$, we can write $m=l_{m} p+m_{0}, n=l_{n} p^{\prime}+n_{0}$ with $0 \leqslant m_{0}<p$ and $0 \leqslant n_{0}<p^{\prime}$. Then,

$$
d=2 \min \left(l_{m}, l_{n}\right) \equiv 2 l
$$

and we can write

$$
R_{m, n}^{\mathrm{tor}}(2 \epsilon)=-\epsilon\left(m \sqrt{-\frac{p^{\prime}}{p}}+\frac{n}{\sqrt{-\frac{p^{\prime}}{p}}}\right) \times \frac{1}{2^{2 l}} \prod_{k=1}^{l} \frac{4 k^{2}-1}{k^{2}}+\mathcal{O}\left(\epsilon^{2}\right)
$$

$l=\min \left(l_{m}, l_{n}\right)$. We need to check that the coefficient also vanishes when $d$ is odd. In that case,

$$
\begin{aligned}
R_{m, n}^{\text {tor }}(2 \epsilon)= & -\epsilon\left(m \sqrt{-\frac{p^{\prime}}{p}}+\frac{n}{\sqrt{-\frac{p^{\prime}}{p}}}+\mathcal{O}(\epsilon)\right) \times\left(\frac{d+1}{2} p b^{2}+\frac{d+1}{2} p^{\prime}-2 \epsilon b\right) \\
& \times \prod_{k=1}^{\frac{d-1}{2}} b^{-1}\left( \pm k p b^{2} \pm k p^{\prime}-2 \epsilon b\right) \times \prod^{\prime}\left(\rho b+\sigma b^{-1}-2 \epsilon\right) \\
& \times \frac{1}{\left(\frac{d+1}{2} p b^{2}+\frac{d+1}{2} p^{\prime}\right) \prod_{k=1}^{\frac{d-1}{2}} b^{-1}\left( \pm k p b^{2} \pm k p^{\prime}\right) \times \prod^{\prime}\left(\rho b+\sigma b^{-1}\right)}
\end{aligned}
$$

Using equation (B.15) we get,

$$
R_{m, n}^{\mathrm{tor}}(2 \epsilon)=-\epsilon\left(m \sqrt{-\frac{p^{\prime}}{p}}+\frac{n}{\sqrt{-\frac{p^{\prime}}{p}}}\right) \frac{d}{2^{d-1}(d+1)} \prod_{k=1}^{\frac{d-1}{2}} \frac{4 k^{2}-1}{k^{2}}+\mathcal{O}\left(\epsilon^{2}\right)
$$

Here $d=2 \min \left(l_{m}, l_{n}\right)-1 \equiv 2 l-1$ which yields,

$$
R_{m, n}^{\mathrm{tor}}(2 \epsilon)=-\epsilon\left(m \sqrt{-\frac{p^{\prime}}{p}}+\frac{n}{\sqrt{-\frac{p^{\prime}}{p}}}\right) \frac{2 l-1}{2^{2 l-1} l} \prod_{k=1}^{l-1} \frac{4 k^{2}-1}{k^{2}}+\mathcal{O}\left(\epsilon^{2}\right)
$$


These terms thus do not contribute in the computation of the character of a physical field. Now we can figure out expressions for the terms in the recursion. The non-vanishing terms that involve $\Delta_{\text {int }}=\Delta_{m, n}^{\epsilon}$ are of the type $\frac{R_{r, s}^{\text {tor }}(2 \epsilon)}{\Delta_{m, n}^{\epsilon}-\Delta_{r, s}}$ with $(m, n) \rightarrow(r, s)_{l}^{ \pm}$Using the regularization of $b$, we have,

$$
\Delta_{m, n}^{\epsilon}-\Delta_{r, s}=( \pm 2 l+1)\left(m \sqrt{-\frac{p^{\prime}}{p}}+\frac{n}{\sqrt{-\frac{p^{\prime}}{p}}}\right) \epsilon+\mathcal{O}\left(\epsilon^{2}\right)
$$

and using expression (4.9) with $\min \left(l_{r}, l_{s}\right)=l-\frac{1}{2} \pm \frac{1}{2}$, we get,

$$
\lim _{\epsilon \rightarrow 0} \frac{R_{r, s}^{\text {tor }}(2 \epsilon)}{\Delta_{m, n}^{\epsilon}-\Delta_{r, s}}=-\frac{1}{2^{2\left(l-\frac{1}{2} \pm \frac{1}{2}\right)}(2 l \pm 1)} \prod_{k=1}^{l-\frac{1}{2} \pm \frac{1}{2}} \frac{4 k^{2}-1}{k^{2}}
$$

The terms involving the extra poles are of the form $\frac{R_{r, s}^{\mathrm{tor}}}{\Delta_{m^{\prime},-n^{\prime}}-\Delta_{r, s}}$ when $\left(m^{\prime},-n^{\prime}\right) \rightarrow(r, s)_{l^{\prime}}^{ \pm}$. We get,

$$
\lim _{\epsilon \rightarrow 0} \frac{R_{r, s}^{\mathrm{tor}}}{\Delta_{m^{\prime},-n^{\prime}}-\Delta_{r, s}}=-\frac{1}{2^{2 l+1} l^{\prime}} \prod_{k=1}^{l} \frac{4 k^{2}-1}{k^{2}}, \quad l=\min \left(l_{r}, l_{s}\right) .
$$

Open Access. This article is distributed under the terms of the Creative Commons Attribution License (CC-BY 4.0), which permits any use, distribution and reproduction in any medium, provided the original author(s) and source are credited.

\section{References}

[1] V. Belavin, X. Cao, B. Estienne and R. Santachiara, Second level semi-degenerate fields in $\mathcal{W}_{3}$ Toda theory: matrix element and differential equation, JHEP 03 (2017) 008 [arXiv: 1610.07993] [INSPIRE].

[2] V. Belavin, B. Estienne, O. Foda and R. Santachiara, Correlation functions with fusion-channel multiplicity in $\mathcal{W}_{3}$ Toda field theory, JHEP 06 (2016) 137 [arXiv: 1602.03870] [INSPIRE].

[3] V.A. Fateev and A.V. Litvinov, On AGT conjecture, JHEP 02 (2010) 014 [arXiv: 0912.0504] [INSPIRE].

[4] B.L. Feigin and D.B. Fuks, Invariant skew-symmetric differential operators on the line and Verma modules over the Virasoro algebra, Funct. Anal. Appl. 16 (1982) 114.

[5] B.L. Feigin and D.B. Fuks, Verma modules over the Virasoro algebra, Funct. Anal. Appl. 17 (1982) 241.

[6] L. Hadasz, Z. Jaskolski and P. Suchanek, Recursive representation of the torus 1-point conformal block, JHEP 01 (2010) 063 [arXiv: 0911.2353] [INSPIRE].

[7] E. Perlmutter, Virasoro conformal blocks in closed form, JHEP 08 (2015) 088 [arXiv: 1502.07742] [INSPIRE].

[8] M. Picco, S. Ribault and R. Santachiara, A conformal bootstrap approach to critical percolation, SciPost 1 (2016) 009. 
[9] M. Picco, S. Ribault and R. Santachiara, A conformal bootstrap solution for critical Potts clusters, in progress.

[10] R. Poghossian, Recursion relations in CFT and $N=2$ SYM theory, JHEP 12 (2009) 038 [arXiv:0909.3412] [INSPIRE].

[11] R. Poghossian, Recurrence relations for the $\mathcal{W}_{3}$ conformal blocks and $\mathcal{N}=2 S Y M$ partition functions, JHEP 11 (2017) 053 [Erratum ibid. 01 (2018) 088] [arXiv: 1705. 00629] [INSPIRE].

[12] S. Ribault and R. Santachiara, Liouville theory with a central charge less than one, JHEP 08 (2015) 109.

[13] S. Ribault, Conformal field theory on the plane, arXiv:1406.4290 [INSPIRE].

[14] Al.B. Zamolodchikov, Conformal symmetry in two dimensions: an explicit recurrence formula for the conformal partial wave amplitude, Commun. Math. Phys. 96 (1984) 419.

[15] Al.B. Zamolodchikov, Conformal symmetry in two-dimensional space: recursion representation of conformal block, Theor. Math. Phys. 73 (1987) 1088. 